\title{
Warenlagergebäude in Deutschland: Eine neue Methodik zur regionalen Quantifizierung der Flächeninanspruchnahme
}

\author{
Daniel Kretzschmar, Robin Gutting, Georg Schiller, Alexandra Weitkamp \\ Eingegangen: 9. Juni 2020 - Angenommen: 27. Januar 2021 - Online veröffentlicht: 26. Februar 2021
}

\begin{abstract}
Zusammenfassung
Rund ein Viertel der neu errichteten Nutzfläche in deutschen Nichtwohngebäuden entfiel im Jahr 2018 auf das Segment der Warenlagergebäude. Trotz dieser großen Bedeutung mangelt es an tiefergehenden Analysen bezüglich der regionalen und sektoralen Bautätigkeit. In der Folge ist bisher wenig über die Flächeninanspruchnahme durch diese Bautätigkeit bekannt. In vorliegendem Beitrag wird eine Methodik zur regionalen Quantifizierung der deutschlandweiten Flächeninanspruchnahme von Warenlagergebäuden vorgestellt. Auf der Basis eines Datensatzes des Forschungsdatenzentrums der Jahre 2000 bis 2015 sowie durch Anwendung GIS-gestützter Umrechnungsparameter sind Aussagen zur kleinräumigen Ausprägung der Flächeninanspruchnahme bis auf Gemeindeebene möglich. Es zeigt sich, dass das Segment der Warenlagergebäude in dreifacher Hinsicht durch Konzentrationseffekte gekennzeichnet ist: Die Gebäude werden immer größer, sie werden in zunehmendem Maße durch spezialisierte Logistikdienst-
\end{abstract}

$\triangle$ Daniel Kretzschmar, Professur für Landmanagement, Technische Universität Dresden, Helmholtzstraße 10, 01069 Dresden daniel.kretzschmar@tu-dresden.de

Robin Gutting, Leibniz-Institut für ökologische Raumentwicklung e.V., Weberplatz 1, 01217 Dresden

r.gutting@ioer.de

Dr. Georg Schiller, Leibniz-Institut für ökologische Raumentwicklung e.V., Weberplatz 1, 01217 Dresden g.schiller@ioer.de

Prof. Dr. Alexandra Weitkamp, Professur für Landmanagement, Technische Universität Dresden, Helmholtzstraße 10, 01069 Dresden

alexandra.weitkamp@tu-dresden.de

(c) 2021 Kretzschmar; licensee oekom verlag. This Open Access article is published under the Creative Commons Attribution-ShareAlike 4.0 International Licence. leister errichtet und konzentrieren sich räumlich auf einige wenige, gut angebundene Standorte. In der Folge nimmt konträr zur allgemeinen Entwicklung die Flächeninanspruchnahme in diesem Segment stetig zu. Für das Gelingen nationaler Flächenhaushaltsziele scheint zukünftig eine differenzierte Betrachtung spezifischer Nutzungsarten, Raumkontexte sowie damit verbundener Einflussgrößen ratsam.

Schlüsselwörter: Warenlagergebäude ·

Nichtwohngebäude · Flächeninanspruchnahme · Methodik ·

räumliche Quantifizierung

\section{Warehouse buildings in Germany: A new methodology for regional land use quantification}

\section{Abstract}

In 2018, the building segment of warehouse buildings accounted for around a quarter of all newly constructed floor space in German non-residential buildings. Despite this great significance, there is a lack of in-depth analyses that look at construction activity in a differentiated manner by region and sector. As a result, little is known so far about the land-use implications of construction in this building segment. This paper a methodology to quantify the land use of warehouse buildings on a regional scale is presented. Based on a data set from the German Research Data Centre for the years 2000 to 2015 and by applying GIS-supported conversion parameters, propositions on the small-scale characteristics of land use are possible down to the municipal level. It is apparent that the segment of warehouse buildings is characterized by concentration effects in three respects: the buildings are getting larger and larger, they are increasingly being constructed by specialized logistics service providers and are spatially con- 
centrated in only a few well-connected locations. As a result, contrary to the general trend, the land use of this segment is steadily increasing. A differentiated consideration of land use along spatial and functional contexts seems to be advisable for the success of national land-use targets.

Keywords: Warehouse buildings - Non-residential buildings · Land use $\cdot$ Methodology $\cdot$ Spatial quantification

\section{Hintergrund}

Der Metabolismus des weltweiten Gebäudebestands verursacht erhebliche Umweltauswirkungen. Bau und Betrieb von Gebäuden sind verantwortlich für rund $36 \%$ des weltweiten Endenergieverbrauchs, 39\% der energiebedingten Kohlendioxidemissionen (IEA 2018: 11), $12 \%$ des Wasserverbrauchs, $40 \%$ des Abfallaufkommens und $40 \%$ des Ressourcenverbrauchs (Becqué/Mackres/Layke et al. 2016: 20). Der Gebäude- und Bausektor ist damit - sowohl hinsichtlich des Endenergieverbrauchs als auch bei den $\mathrm{CO}_{2-}$ Emissionen - der weltweite Hauptverursacher, noch vor dem Transportsektor und der Industrie. Zwischen 2010 und 2016 stieg die global in Gebäuden errichtete Nutzfläche um rund 15\% (IEA 2018: 11) auf 235 Milliarden $\mathrm{m}^{2}$ (UN/IEA 2017: 7). Bis 2060 wird sich nach Schätzungen der Vereinten Nationen dieser Bestand nochmals verdoppelt haben mit deutlichen Folgen für die Ressourcen- und Flächeninanspruchnahme (UN/IEA 2017: 13).

In vielen europäischen Staaten entfällt rund die Hälfte der Nutzfläche aller Gebäude auf den Teilmarkt der Nichtwohngebäude (Kohler/Steadman/Hassler 2009: 451). Ortlepp, Gruhler und Schiller (2016: 853) legen vergleichbare Schätzungen für Deutschland vor. Trotz der dementsprechend großen Bedeutung des Nichtwohngebäudebestandes für die Umsetzung klima- und ressourcenschutzpolitischer Zielstellungen mangelt es an räumlich und sachlich differenzierten Informationen $\mathrm{zu}$ dieser Nutzungsart (Bradley/Kohler 2007; Hassler 2009). Der Nichtwohngebäudebestand ist sehr heterogen. In ihm sind Gebäude der Daseinsvorsorge ebenso enthalten wie Industriegebäude, Bürogebäude oder Logistikgebäude. Ohne umfassende Informationen zu diesen Nutzungsklassen - ,wo nicht einmal die Anzahl der Produktions-, Handels-, Lager-, Büro und Verwaltungsgebäude, Hotels, Gaststätten etc. verfügbar ist““ (Rein 2016: 10) - kann eine gezielte umweltpolitische Steuerung nicht erfolgen. Als einer der wenigen Staaten weltweit weist Großbritannien nutzungsklassenbezogene Informationen zum nationalen Nichtwohngebäudebestand aus. Es gilt damit als das Land mit dem größten Kenntnisstand des eigenen Nichtwohngebäudebestandes (Isaacs/Hills 2014: 96). Nach Berechnungen des britischen „Depart- ment for Business, Energy and Industrial Strategy“ (DBEIS 2016: 13) entfällt für England und Wales der größte Anteil der Gesamtnutzfläche in Nichtwohngebäuden auf Industriegebäude $(22 \%)$, gefolgt von Warenlagergebäuden (18\%), Bürogebäuden (15\%) und Handelsgebäuden (14\%).

In Deutschland existiert bis heute keine amtliche Statistik zu Größe, Struktur und Verteilung des Nichtwohngebäudebestands (Rein 2016: 3). Lediglich die jährliche Bau- und Abrisstätigkeit wird nutzungsklassenspezifisch erfasst. So wurden im Jahr 2018 rund 25,7 Millionen m $^{2}$ Nutzfläche in Nichtwohngebäuden neu errichtet. Die Nutzungsklassen mit den größten Anteilen am jährlichen Zuwachs sind hierbei die Warenlagergebäude (26\%) sowie die Industriegebäude (17\%), gefolgt von landwirtschaftlich genutzten Gebäuden (13\%) (Statistisches Bundesamt 2020: 20 ff.).

Sowohl bezüglich des Bestandes als auch im Hinblick auf die Dynamik zeigt sich der hohe Stellenwert der Warenlagergebäude als wesentlicher Teilmarkt der Nichtwohngebäude. Als Warenlagergebäude gelten gemäß Definition des Statistischen Bundesamtes ,Nichtwohngebäude, die für die Lagerung von Waren aller Art bestimmt sind" (Statistisches Bundesamt 2010: 10). Trotz des hohen Stellenwerts wurden Warenlagergebäude bis zum Jahr 2014 durch das Statistische Bundesamt nicht als eigenständige Nutzungsklasse geführt, sondern kumuliert mit den Handelsgebäuden ausgewiesen (Kretzschmar/Schiller/Weitkamp 2019:164). Eine tiefergehende Analyse der Bestandsveränderung war auf dieser Grundlage ausgeschlossen. Daten zur bundesweiten Bautätigkeit im Nichtwohnbau durch das Forschungsdatenzentrum (FDZ) ermöglichen dagegen eine umfassende Auswertung auch für Warenlagergebäude. Aufbauend auf diesem Datensatz möchte der Beitrag im Folgenden die Bautätigkeit von Warenlagergebäuden in Deutschland vor dem Hintergrund der hierdurch induzierten Flächeninanspruchnahme analysieren. Fokus der Analyse ist die Frage, welche Ursachsen dieser hohen Flächendynamik zugrunde liegen und in welchen Räumen diese Dynamik besonders stark zum Flächenwachstum beiträgt. Basis der Analyse bildet eine neue Quantifizierungsmethode, welche kleinräumige Forschungsdaten der Bautätigkeitsstatistik mit GIS-gestützten Umrechnungsparametern kombiniert.

Im Folgenden wird nach einer Einordnung des Themas in die Forschungslandschaft (Kapitel 2) der methodische Ansatz zur Quantifizierung der Flächeninanspruchnahme beschrieben (Kapitel 3). Anschließend werden die hieraus abgeleiteten Befunde gebäudegrößenbezogener, sektoraler und räumlicher Konzentrationsprozesse im Warenlagersegment präsentiert (Kapitel 4 und 5). Nach einer kritischen Einordnung des methodischen Vorgehens sowie der hieraus gewonnenen Ergebnisse (Kapitel 6) schließt der Beitrag mit einem Fazit zur Flächeninanspruchnahme von Warenlagergebäuden in Deutschland (Kapitel 7). 


\section{Bedeutungszuwachs von Warenlagergebäuden}

Warenlagergebäude stehen in den letzten Jahren verstärkt im Fokus des wissenschaftlichen Erkenntnisinteresses (He/ Shen/Wu et al. 2018: 7). Ein wesentlicher Treiber dieses gesteigerten Interesses gründet auf der drastischen Zunahme der weltweiten Warenströme im Zuge der Globalisierung (Hall/Hesse 2013: 4). Darüber hinaus ist das Aufkommen des Online-Handels ein wesentlicher Treiber des Strukturwandels (Kienzler/Altenburg/Esser et al. 2018). Infolge des steigenden Anteils des E-Commerce wächst die Nachfrage nach Gebäuden zur vorrätigen Lagerung, Verpackung und Verteilung von im Internet erworbenen Produkten (Murphy 2003). Zwischen 1996 und 2016 hat sich das Sendungsvolumen von Kurier-, Express- und Paketsendungen in Deutschland von jährlich 1,4 Mrd. Sendungen auf rund $3 \mathrm{Mrd}$. Sendungen mehr als verdoppelt, während sich der Umsatz im gleichen Zeitraum fast verdreifachte (Manner-Romberg/Müller-Steinfahrt 2017: 25 f.). Beide Entwicklungen - sowohl das Aufkommen des Online-Handels als auch die Professionalisierung der Warenströme infolge der Globalisierung - verändern die Anforderungen an moderne Warenlagergebäude.

Zur Überwindung nationaler Grenzen sowie zur Etablierung global verschränkter wirtschaftlicher Warenströme wurde ein neues, komplexeres Netzwerk von Verkehrsknotenpunkten, sogenannter Hubs, notwendig (Hesse/Rodrigue 2004: 174). Kleinere, ältere Warenlager können aufgrund ihrer Bauart den betrieblichen Anforderungen an eine moderne Logistik nicht gerecht werden (Aljohani/Thompson 2016: 3). Statt wie bisher die Lagerhaltung zwischen Herstellern und Empfängern der Waren zu verteilen, wird diese in zunehmendem Maße durch wenige nationale und regionale Logistikzentren organisiert, die ein weitaus größeres geographisches Gebiet bedienen können (Allen/Browne/ Cherrett 2012: 46). Im Zuge der Zentralisierung der Logistik sowie technischer Fortschritte bei Lagerung und Abfertigung von Produkten sind Gebäude mit weitaus größeren Lagerflächen vorteilhaft (Green Leigh/Hoetzel 2012: 88). Prototypen dieser neuen Warenlagergebäude sind die Distributionsimmobilien (Baker 2004): große, 24 Stunden am Tag betreibbare Zentrallager (Klauser 2012: 87). Die klassische Lagerfunktion wurde in diesen Gebäuden mehr und mehr um Sortierungs-, Kommissionierungs-, Verpackungsund Verteilungsfunktionen ergänzt und erweitert, um den gesteigerten zeitlichen Ansprüchen in einem nachfragegetriebenen Logistikumfeld gerecht werden zu können. Während Gebäude mit klassischen Lagerfunktionen zum Teil sehr hohe Deckenhöhen aufweisen, um die Produktivität des Gebäudes zu maximieren (Hochregallager), benötigen Distributionsimmobilien - in denen ein großer Teil des
Durchsatzes innerhalb eines Tages oder einiger Stunden erfolgt - erhebliche Bereitstellungsflächen im Erdgeschoss (Bowen 2008: 380).

Wesentliche Voraussetzungen für die Errichtung eines Warenlagers ist das Vorhandensein einer gut ausgebauten Transportinfrastruktur (Kumar/Zhalnin/Kim et al. 2017) wobei die Nähe zu Autobahnen, Flug- und Seehäfen alle anderen Infrastrukturen bei Weitem an Bedeutung übersteigt (Bowen 2008: 386; Woudsma/Jensen/Kanaroglou et al. 2008: 295; Verhetsel/Kessels/Goos et al. 2015: 119). Hinzu kommt ein möglichst naher Absatzmarkt für die in ihnen prozessierten Waren (Cidell 2010: 367), die Verfügbarkeit von Logistikarbeitskräften und Flächen (Nehm/ Veres-Homm 2018: 11) sowie niedrige Bodenpreise (Jakubicek/Woudsma 2011: 168; Verhetsel/Kessels/Goos et al. 2015: 119). In der Kombination dieser Faktoren lassen sich zwei charakteristische Standorttypen von Warenlagergebäuden identifizieren: Standorte ,auf der grünen Wiese' mit geringer Flächenkonkurrenz und attraktiven Bodenpreisen in der Nähe großer Agglomerationen sowie Standorte an Verkehrsknotenpunkten zur Minimierung von Lieferzeiten und -kosten (Cidell 2015).

Großstädte mit ihrer generell gut ausgebauten Transportinfrastruktur und hohen Bevölkerungsdichte sind in diesem Zusammenhang von besonderem Interesse für die Ansiedlung neuer Warenlagergebäude. Hierbei lassen sich zwei Entwicklungen feststellen: die systematische Verdrängung großer Warenlagergebäude aus den Kernstädten an die suburbanen Ränder als Folge massiv gestiegener Bodenpreise (logistics sprawl) (Dablanc/Ross 2012) sowie das zeiteffizienzgetriebene Bestreben der Logistikdienstleister, dieser Verdrängung durch die Etablierung kleinerer Warenlagergebäude in den Städten zu begegnen (city logistics) (Bulwiengesa 2017: 74).

Durch die fortschreitende Globalisierung, wachsende Waren- und Paketströme und die Etablierung gewaltiger logistischer Cluster - insbesondere in den suburban-zersiedelten Umlandregionen großer Städte - wuchsen in den letzten Jahren „Landschaften der Logistik [...]. Diese Landschaften dürften zu den bedeutsamsten Veränderungen zählen, die es während der letzten Jahrzehnte in der gebauten Umwelt gegeben hat" (Waldheim/Berger 2012: 77) (vgl. Abbildung 1). An diesen Standorten ist ein ausgedehntes Flächenwachstum zu beobachten, und zwar nicht nur in Bezug auf die reinen Gebäudegrundflächen der Warenlager, sondern auch hinsichtlich der damit einhergehenden Verkehrs-, Verlade-, Pack- und Parkflächen (Klauser 2012). Hinzu kommt der Einfluss des durch diese Gebäude induzierten Lieferverkehrs: Licht-, Luft- und Wasserverschmutzung, eine übermäßige Beanspruchung der Transportinfrastruktur, Staus, Vibrationserschütterungen und Lärmbelästigung sind die Folgen (Jenks/Jencks/Rogers et al. 


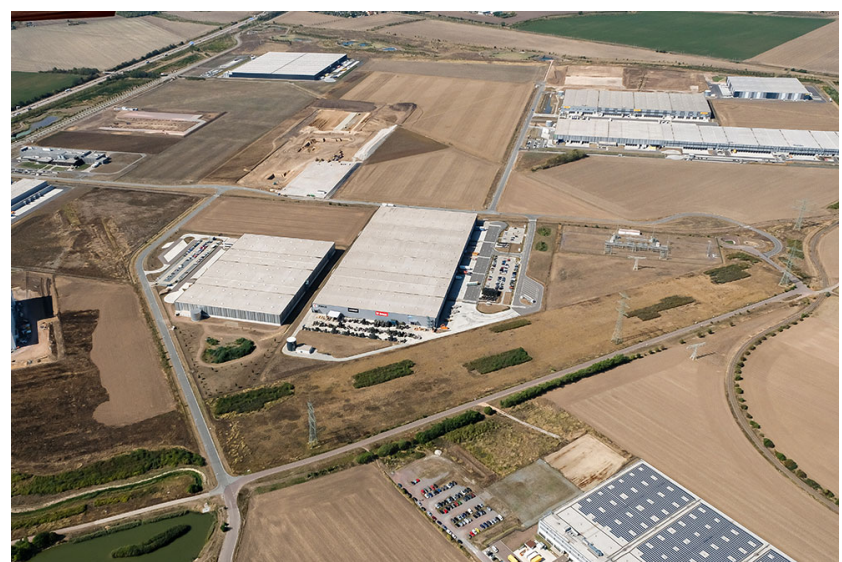

Abbildung 1 Baustelle zum Neubau eines Logistikzentrums im Stadtteil Peißen in Halle (Saale) (2019)

Quelle: euroluftbild.de/Maike Glöckner

2012: 5). Die Auswirkungen auf die Flächeninanspruchnahme konnten bisher durch die Forschung nicht angemessen untersucht werden (Aljohani/Thompson 2016: 5 f.). Ein wesentlicher Grund hierfür ist das Fehlen amtlicher Daten, wodurch die Quantifizierung und Verortung von Warenlagergebäuden und deren Flächenverbrauch in Deutschland deutlich erschwert ist.

Das Statistische Bundesamt subsummiert eine Vielzahl unterschiedlicher Gebäudetypen unter dem Begriff der Warenlagergebäude. Hierzu zählen Logistikzentren, Versandhäuser, Kühl- und Lagerhäuser, aber auch Speicher, Silos, Kraftstofflager und Frachtguthallen sowie jegliche Lagergebäude staatlicher Bevölkerungsschutzinstitutionen (unter anderem Feuerwehr, Polizei, Bundesgrenzschutz) (Statistisches Bundesamt 2010: 10f.). Das Verständnis des Statistischen Bundesamts ist deutlich breiter gefasst als jenes immobilienwirtschaftlicher Akteure, welche sich auf den (privatwirtschaftlichen) Bestand der Logistikzentren, Versandhäuser und klassischen Lagerhäuser konzentrieren. So klassifiziert Bulwiengesa (2018) Logistikimmobilien in Distributionsimmobilien, E-Fulfillment-Center ${ }^{1}$, Paketverteilzentren, Zustellbasen, Produktions-, Hochregal- und Kühllager. Trotz dieser großen definitorischen Breite der Bundesstatistik findet eine Ausweisung entlang bestimmter Warenlagergebäudetypen nur sehr eingeschränkt statt. Das Statistische Bundesamt kennt entsprechend des aktuellen Signierschlüssels des Jahres 2014 nur „Warenlagergebäude für die öffentliche Nahrungsmittelvorsorge“, „Warenlagergebäude der Polizei, des Bundesgrenz-, Feuer- und zivilen

\footnotetext{
1 Unter diesem Typ werden Warenlager verstanden, welche Kommissionierung, Verpackung, Versand und Retourenmanagement von im Onlinehandel erworbenen Waren schnellstmöglich abwickeln.
}

Bevölkerungsschutzes (auch Bundespolizei)“ sowie „Andere Warenlagergebäude“, wobei in der letzten Kategorie alle anderweitig genutzten Warenlagergebäude unabhängig von Größe und Funktion, dem Nutzer oder Eigentümer sowie dem Gebäudetyp zusammengefasst wurden (Statistisches Bundesamt 2014).

Die Forschung ist aus diesem Grund auf Erhebungen von Maklern, Immobilienberatern, Investoren und Projektentwicklern angewiesen. Zwar hat die Zahl immobilienwirtschaftlicher Studien, Marktberichte und Standortreports zu dieser Nutzungsklasse in den letzten Jahren deutlich zugenommen (Kille/Nehm 2018: 21), dennoch stehen bislang keine grundsätzlichen Informationen beispielsweise zur Häufigkeit und Größe des Bestandes und seiner Verteilung im Raum zur Verfügung. Unterschiedliche Initiativen der Immobilienforschung widmen sich dem Aufbau eigener Erhebungen und Datenbanken (z. B. riwis-Datenbank, Fraunhofer IIS, IndustrialPort). Diese sind bezüglich der verwendeten Definitionen, den zugrunde gelegten Erhebungsteilräumen sowie der betrachteten Erhebungsgröße (Nutzfläche, Flächenumsätze, Mietniveaus) nicht vergleichbar. Somit „bleibt dieser Immobilientyp bezüglich Marktdaten und neutralen Standortinformationen ein vergleichsweise wenig bearbeitetes Feld" (Veres-Homm/Kübler/Weber et al. 2015: 20). Um Erkenntnisse über Ursachen und räumliche Auswirkungen zu gewinnen, wird im folgenden Kapitel ein methodisches Verfahren zur deutschlandweiten Quantifizierung der Baumaßnahmen im Segment der Warenlagergebäude sowie der hieraus folgenden Flächeninanspruchnahme vorgestellt.

\section{Methodik}

Das methodische Vorgehen zur Ermittlung der Flächeninanspruchnahme von Warenlagergebäuden präsentiert das Fließdiagramm in Abbildung 2. Grundlage dieses Beitrags ist ein Datensatz des Forschungsdatenzentrums (FDZ) des Bundes und der Länder von 2018 zur Bautätigkeit der Jahre 2000 bis 2016. ${ }^{2}$ Das Forschungsdatenzentrum stellt mit diesem Datensatz Zahlen zu Baufertigstellungsstatistiken für alle Bundesländer harmonisiert und zusammengefasst zur Verfügung. Er enthält Informationen zu Art und Umfang aller in Deutschland in diesem Zeitraum der Statistik bekannten Baufertigstellungen von Wohn- und Nichtwohngebäuden, wobei Abweichungen von den Zahlen der amtlichen Statistik infolge eines angewandten Anonymisierungsverfahrens nicht auszuschließen sind. Neben Informationen

\footnotetext{
2 http://www.forschungsdatenzentrum.de/de/bauen-undwohnen/baufertigstellungen (21.12.2020).
} 


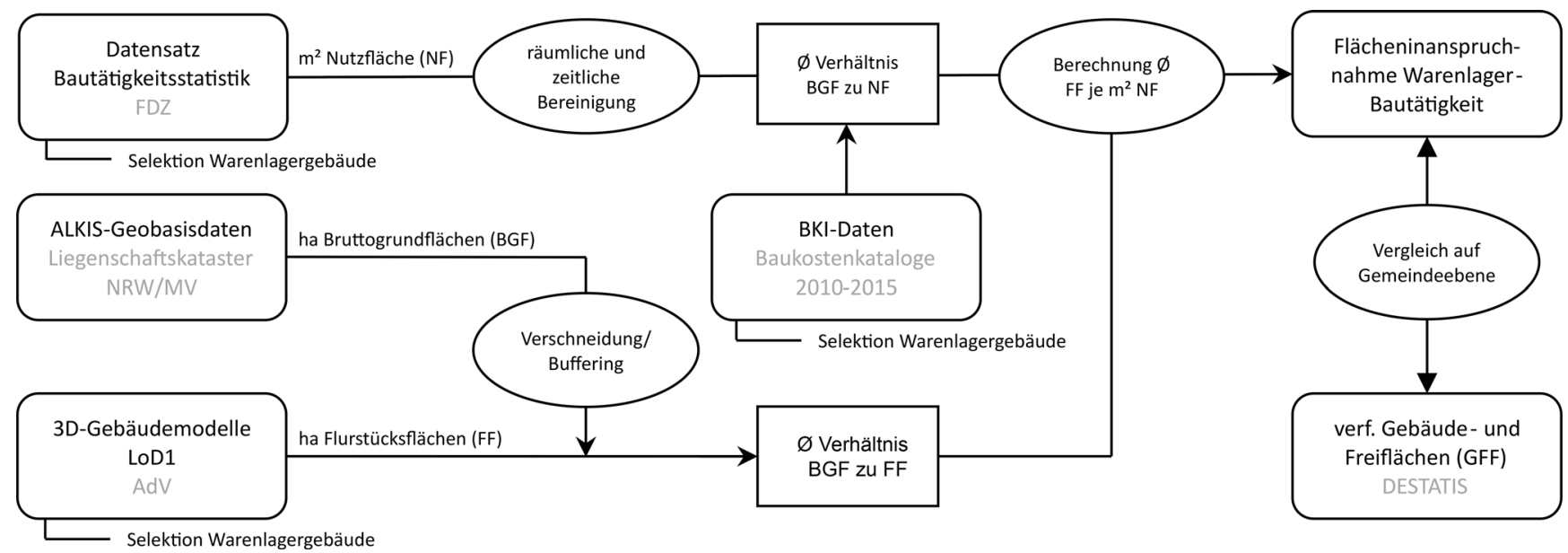

Abbildung 2 Methodisches Vorgehen zur Ermittlung der Flächeninanspruchnahme der Bautätigkeit von Warenlagergebäuden Anmerkungen: Die Datenquellen sind in grauer Schrift angegeben. Abkürzungen: FDZ = Forschungsdatenzentrum des Bundes und der Länder, ALKIS = Amtliches Liegenschaftskatasterinformationssystem, NRW = Nordrhein-Westfalen, MV = Mecklenburg-Vorpommern, AdV = Arbeitsgemeinschaft der Vermessungsverwaltungen der Länder der Bundesrepublik Deutschland, DESTATIS = Statistisches Bundesamt

zur Größe des Gebäudezugangs (in $\mathrm{m}^{2}$ Wohn- bzw. Nutzfläche) sind unter anderem Angaben zur Art des Nichtwohngebäudes nach 52 (Statistisches Bundesamt 2010) bzw. 73 Nutzungstypen (Statistisches Bundesamt 2014), zum Bauherren, zur Lage des Gebäudes (Land, Kreis, Gemeinde), zur Gebietsgrößenklasse nach Einwohnern sowie zum Zeitpunkt der Baufertigstellung und zum Zeitpunkt der Erfassung in der Statistik enthalten. Insgesamt sind in diesem Datensatz rund 3,8 Millionen einzelne Baumaßnahmen verzeichnet, wobei Warenlagergebäude hiervon rund 116.000 Fälle ausmachen.

Bezüglich des Zeitpunkts der Erfassung in der Statistik und des tatsächlichen Zeitpunkts der Baumaßnahme sind Abweichungen möglich. Häufig halten Baumaßnahmen erst ein oder zwei Jahre nach der tatsächlichen Fertigstellung Einzug in die Statistik. In wenigen Extremfällen erfolgte die tatsächliche Fertigstellung mehrere Jahrzehnte vor Erfassung in der Statistik. Rund 5\% der Nutzfläche eines Jahrgangs sind erst in nachfolgenden Berichtsjahren in der Statistik verzeichnet. Da sich der Datensatz stets auf den Zeitpunkt des Eingangs in die Statistik bezieht, waren zeitliche Bereinigungen notwendig, um einen Datensatz zu erzeugen, welcher kohärent auf den Zeitpunkt der tatsächlichen Baumaßnahme orientiert. ${ }^{3}$ Das aktuellste Berichtsjahr 2016 kann nicht um nachträglich gemeldete Fertigstellungszahlen

\footnotetext{
3 Der Datensatz des Forschungsdatenzentrums enthält beide Zeitinformationen: den Zeitpunkt des Eingangs in die Statistik sowie den tatsächlichen Fertigstellungszeitpunkt der Baumaßnahme. Im Datensatz waren die Baumaßnahmen entlang des Zeitpunkts des Eingangs in die Statistik den Jahresscheiben zugeordnet und sortiert. Diese Systematik wurde auf den Zeitpunkt der tatsächlichen Fertigstellung hin angepasst.
}

aktualisiert werden und wird aus diesem Grund in der nachfolgenden Analyse nur zur graphischen Trendabschätzung berücksichtigt. Es ist nicht Gegenstand der Berechnungen.

Neben der zeitlichen Bereinigung ist auch eine Prüfung der inhaltlichen Konsistenz der Signierschlüssel erforderlich, da das Signierschlüsselverzeichnis innerhalb der Zeitreihe durch die Bundesstatistik verändert wurde. Hierfür wurde das jüngere und umfangreichere Verzeichnis von 72 Nutzungstypen auf das ältere Verzeichnis mit 52 Nutzungstypen übertragen. Für die hier betrachteten Warenlagergebäude sind die Kategorien beider Verzeichnisse identisch. Die inhaltliche Prüfung zeigte, dass sachliche Informationen zum Teil Fehlwerte aufwiesen. Da nicht alle Fälle korrekt mit der jeweiligen Gebietsgrößenklasse (nach Einwohnern) versehen waren, wurden diese Informationen ergänzt. Für die weiterführende Analyse wird der Typ „Andere Warenlagergebäude“ selektiert.

Zusätzlich zur sachlichen und zeitlichen Anpassung sind auch die in der Statistik enthaltenen räumlichen Informationen über den Zeitverlauf nicht kohärent. Eingemeindungen, Gebietsänderungen sowie Kreis- und Gemeindegebietsreformen sorgen dafür, dass sich das Verzeichnis der Amtlichen Gemeindeschlüssel (AGS) mit jedem Jahr ändert. Um zu einem Datensatz mit einheitlichem Gebietsstand zu gelangen, wurden alle Fälle von Warenlagergebäuden in der Baufertigstellungsstatistik mit einer Nutzflächenänderung von über $2.500 \mathrm{~m}^{2}$ auf den Gebietsstand des Jahres 2015 übertragen. Durch Setzung einer Bagatellgrenze von mindestens $2.500 \mathrm{~m}^{2}$ Nutzfläche je Baumaßnahme verringerte sich die Zahl der zu berücksichtigenden Fälle um rund $94 \%$, wodurch der Bereinigungsaufwand insbesondere hinsichtlich der amtlichen Gemeindeschlüssel deutlich abnimmt. Trotz dieser deutlichen Bereinigung berücksichtigt der Datensatz 
noch immer rund $53 \%$ der im Gesamtzeitraum in Deutschland errichteten Warenlagernutzfläche. Auch Veres-Homm, Kübler, Weber et al. (2015: 24) beziehen sich bei ihrer Erhebung deutscher Logistikimmobilien auf Gebäude über $2.500 \mathrm{~m}^{2}$

Alle Fälle der vorangegangenen 15 Jahre wurden hinsichtlich der mit ihnen verknüpften AGS-Information mit dem Verzeichnis des Jahres 2015 abgeglichen und bei Übereinstimmung zugeordnet. Bei fehlender Übereinstimmung (infolge der oben beschriebenen Gebietsänderungen) wurden die Fälle auf den jeweils neu gültigen Gemeindeschlüssel übertragen. Gebietsveränderungen konnten demnach nur bezüglich der Signierung über den amtlichen Gemeindeschlüssel berücksichtigt werden. Tatsächliche Verlagerungen des Standorts einer Baumaßnahme bleiben in Ermangelung exakter Standortinformationen unberücksichtigt.

Der bereinigte Datensatz enthält rund 6.700 Fälle von Warenlager-Baumaßnahmen. Als Baumaßnahmen werden sowohl Neubaumaßnahmen als auch Maßnahmen im Bestand (Umbau, Ausbau, Umwidmung) verstanden. Gleichzeitig sind es genau diese Warenlagergebäude, welche hinsichtlich ihrer Standortwahl und der durch sie verursachten Flächeninanspruchnahme von besonderem Interesse sind. Bei kleineren Lagern wird bezogen auf die Verteilung im Raum ein eher zufälliges Bild erwartet. Sie werden weit weniger stark von Industrie- oder Dienstleistungsunternehmen, sondern vielmehr von privaten Haushalten, Handwerksbetrieben und Selbstständigen errichtet und stehen nicht im Fokus der hier vorgestellten Untersuchungen.

Nach der Bereinigung wurden die im Datensatz erfassten Nutzflächen in Bruttogrundflächen umgerechnet. Grundlage hierfür waren die zwischen 2010 und 2015 durch das Baukosteninformationszentrum Deutscher Architektenkammern (BKI 2010; BKI 2012; BKI 2013; BKI 2015) veröffentlichten Objektdaten von 32 deutschlandweit erhobenen Warenlagergebäuden. ${ }^{4}$ Diese enthalten Informationen unter anderem zur Nutz- und Bruttogeschossfläche der Gebäude. Im Warenlagersegment sind Gebäude mit mehr als einem Stockwerk sehr selten, weshalb die Bruttogeschossfläche der BKI-Objekte unverändert auf die Bruttogrundfläche übertragen wurde. Unter dieser Annahme wurde das objektbezogene Verhältnis von Nutzfläche zu Bruttogrundfläche

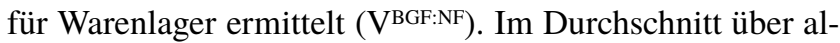
le Objekte führt $1 \mathrm{~m}^{2}$ Nutzfläche zu einer Bruttogrundfläche von rund $1,2 \mathrm{~m}^{2}$. Hierbei ist es unerheblich, ob es sich um große oder kleine Warenlagergebäude handelt.

\footnotetext{
${ }^{4}$ Das BKI unterscheidet diese in „Lagergebäude ohne Mischnutzung“, „Lagergebäude mit bis zu $25 \%$ Mischnutzung“ und „Lagergebäude mit mehr als $25 \%$ Mischnutzung“. In die Berechnung sind alle drei Objektarten zu etwa gleichen Teilen eingeflossen.
}

Um die gesamte Flächeninanspruchnahme einschließlich der umgebenden Flurstückflächen erfassen zu können, wurde in einem nächsten Schritt das durchschnittliche Verhältnis aus Bruttogrundfläche und umgebenden Flurstückflächen von Warenlagergebäuden ermittelt. Grundlage hierfür bildeten Geobasisdaten aus acht Kreisen der Länder Nordrhein-Westfalen und Mecklenburg-Vorpommern. ${ }^{5}$ Zum einen sind in diesen Bundesländern Geobasisdaten für wissenschaftliche Einrichtungen offen und kostenfrei zu beziehen (open data). Zum anderen gewährleistet die Auswahl eines dicht besiedelten Flächenlandes sowie eines dünn besiedelten, ländlich geprägten Bundeslandes die Generalisierbarkeit der Ergebnisse auf das gesamte Bundesgebiet. Um innere Unterschiede unterschiedlicher Siedlungsstrukturen überprüfen zu können, wurden stellvertretend für die vier Kreistypen des Bundesinstituts für Bau-, Stadt- und Raumforschung (BBSR) (Kreisfreie Großstädte, Städtische Kreise, ländliche Kreise mit Verdichtungsansätzen, dünn besiedelte ländliche Kreise) ${ }^{6}$ jeweils zwei Landkreise analysiert. Innerhalb dieser wurden alle Warenlagergebäude detektiert und mit ihren jeweiligen Flurstücken verschnitten.

Basis für die Ermittlung der Gebäudegrundflächen bildete ein aufbereiteter Lod1-Datensatz ${ }^{7}$ des Leibniz-Instituts für ökologische Raumentwicklung (IÖR), in welchem 3DGebäudemodelle in 2D-Flächeninformationen umgewandelt wurden. In diesen Daten ist die Nutzungsart der Gebäude vermerkt, wodurch die Zuordnung zu Warenlagergebäuden möglich wird. Zur Ermittlung der Flurstïckflächen wurden Geobasisdaten der Liegenschaftskataster der betreffenden Bundesländer herangezogen. Zunächst wurden innerhalb der Kreisgrenzen alle Gebäude des 2DFlächeninformations-Datensatzes selektiert. Anschließend wurde die Auswahl auf jene Gebäude begrenzt, die eine Nutzung als Warenlagergebäude aufwiesen. ${ }^{8}$ In den 2DFlächeninformationsdaten getrennt erfasste Gebäudegrund-

\footnotetext{
${ }^{5}$ Betrachtet wurden die Kreise Unna, Rhein-Kreis Neuss, Hochsauerlandkreis, Nordwestmecklenburg, Ludwigslust-Parchim, Vorpommern-Rügen sowie die kreisfreien Städte Köln und Rostock.

${ }^{6}$ https://www.bbsr.bund.de/BBSR/DE/forschung/ raumbeobachtung/Raumabgrenzungen/deutschland/ kreise/siedlungsstrukturelle-kreistypen/kreistypen.html (21.12.2020).

7 In diesem Level-of-Detail-1-Datensatz werden 3D-Gebäudemodelle stark vereinfacht als grundflächenbezogene Klötzchen ohne Dach und Texturen dargestellt.

${ }^{8}$ Entsprechend der Systematik der Arbeitsgemeinschaft der Vermessungsverwaltungen der Länder der Bundesrepublik Deutschland (AdV) als Warenlagergebäude erfasst wurden Gebäude der Funktionsbezeichnung Kühlhaus, Gebäude für Vorratshaltung, Speichergebäude, Lagerhalle, Lagerschuppen, Lagerhaus sowie Speditionsgebäude.
} 


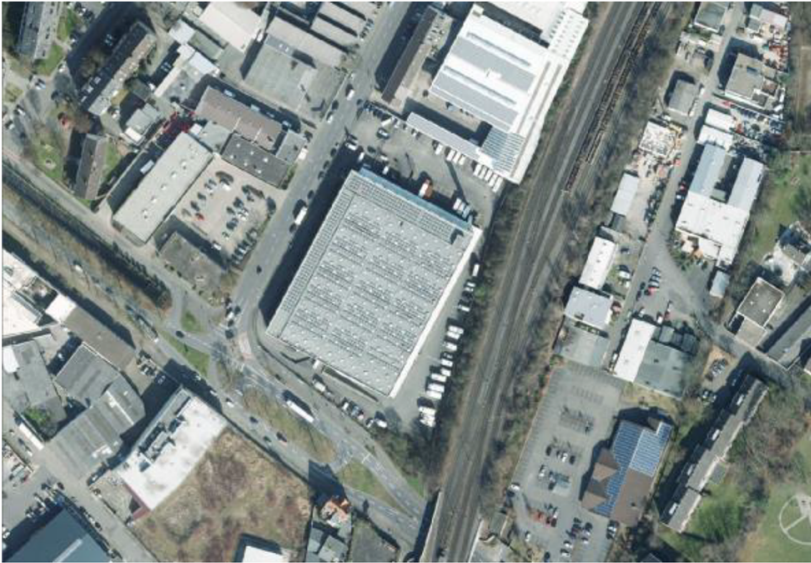

1. Beispiel-Gebiet

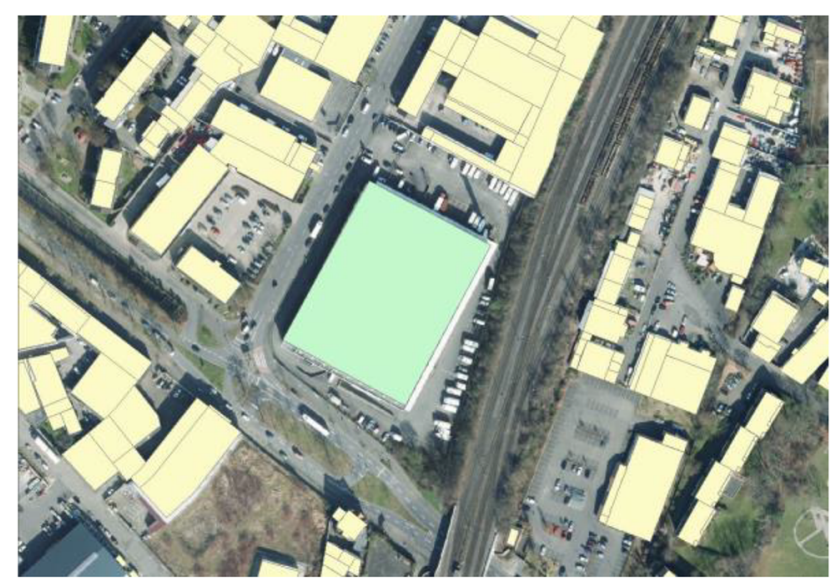

3. Selektion von Warenlagergebäuden

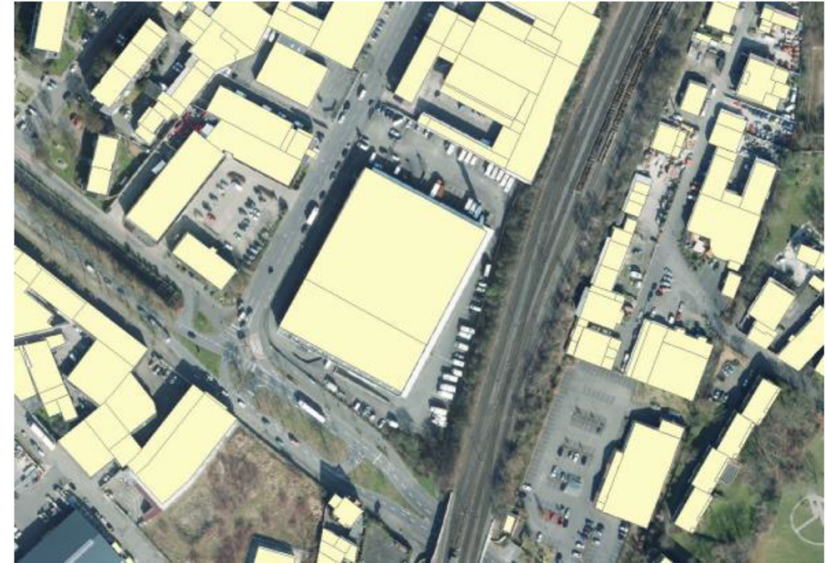

2. Zusammenfassung von 2D-Gebäudegrundrissen

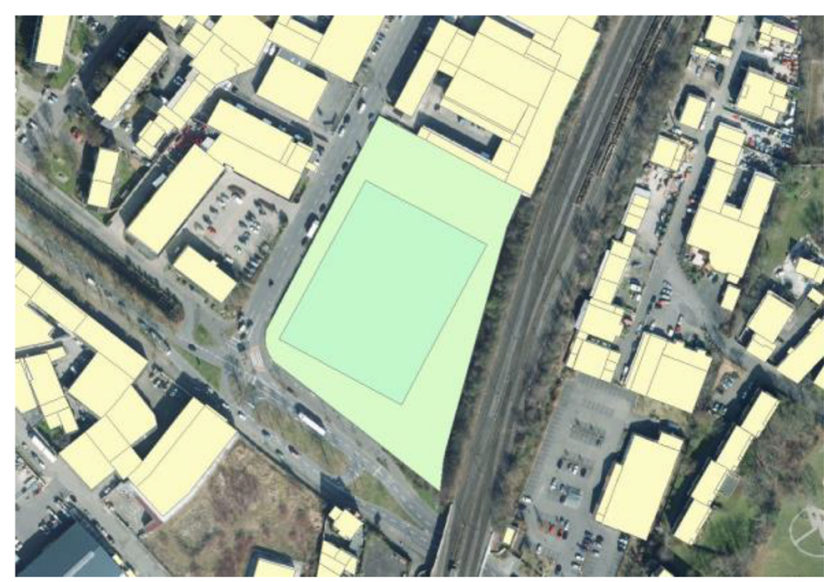

4. Berechnung des Flächen-Buffers

Abbildung 3 Beispielhafte Visualisierung der GIS-Berechnungsschritte an einem Warenlager in Nordrhein-Westfalen Bildquelle: Daniel Kretzschmar, IÖR.

risse wurden im Falle sich tangierender Geometrien und gleicher Gebäudefunktionen zusammengefasst.

Schlussendlich wurden jene Gebäude betrachtet, welche Gebäudegrundrisse mit einer Fläche von größer $3.000 \mathrm{~m}^{2}$ aufwiesen und gleichzeitig im Datensatz des Amtlichen Liegenschaftskatasterinformationssystems (ALKIS) innerhalb von Flurstücken der tatsächlichen Nutzung „Industrie und Gewerbe" verortet waren (entspricht der Bruttogrundfläche BGF)..$^{9}$ Insgesamt konnten 436 Warenlagergebäude dieser Größenklasse in den acht Kreisen identifiziert werden, die vollständig zur weiteren Berechnung herangezogen wurden. Radial um diese Gebäudegrundrisse wurde in ei-

\footnotetext{
9 Der Grenzwert von $3.000 \mathrm{~m}^{2}$ ergibt sich aus der vorherigen Bagatellgrenze von $2.500 \mathrm{~m}^{2}$ Nutzfläche multipliziert mit dem Verhältnis $\mathrm{V}^{\mathrm{BGF}: \mathrm{NF}}$ von 1,2 .
}

nem 50-Meter-Radius ein Buffer ${ }^{10}$ erzeugt, der wiederum mit den umgebenden Flurstückgrenzen, Gebäuden anderer Nutzung sowie Warenlagergebäuden kleiner $3.000 \mathrm{~m}^{2}$ verschnitten wurde (entspricht der Flurstückfläche FF). Hierdurch wurde gewährleistet, dass Flächen anderer Flurstücke sowie Straßen und andere Gebäude innerhalb des gleichen Flurstücks um das Warenlager bei der Berechnung der Flächeninanspruchnahme unberücksichtigt blieben und lediglich die rein durch das Warnlagergebäude

\footnotetext{
10 Der Anwendung eines 50-Meter-Puffers liegt die Annahme zugrunde, dass in engen Märkten mit hoher Flächenknappheit bereits vor Erreichen des 50-Meter-Buffers die Flurstückfläche durch die verschnittenen Flurstückgrenzen definiert wird. Gleichzeitig gewährleistet ein 50-Meter-Buffer eine ausreichende Erfassung größerer Freiflächen für Schütt- und Massengutlagerung in Hafenstandorten (z. B. Rostock, Stralsund, Wismar).
} 


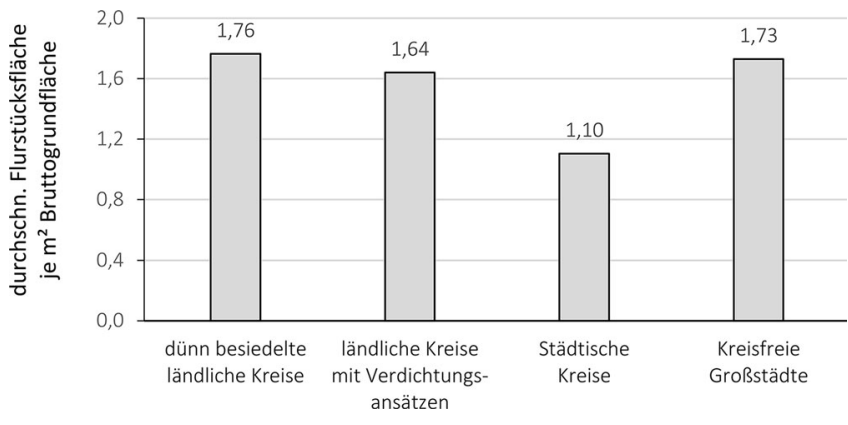

Abbildung 4 Durchschnittliche umgebende Flurstückfläche je 1 $\mathrm{m}^{2}$ Bruttogrundfläche der Warenlagergebäude, bezogen auf siedlungsstrukturelle Kreistypen

induzierte Flurstïckfläche verblieb. In Abbildung 3 sind die beschriebenen Arbeitsschritte exemplarisch abgebildet. Abschließend wurden die Bruttogrundflächen sowie die umgebenden Flurstückflächen aller Objekte innerhalb eines Landkreises kumuliert und ins Verhältnis gesetzt. Im Ergebnis lässt sich für alle betrachteten Landkreise das Verhältnis von Bruttogrundflächen zu Flurstückflächen (VBGF:FF) für Warenlagergebäude ermitteln.

In Abbildung 4 sind diese Verhältnisse entsprechend der Systematik siedlungsstruktureller Kreistypen des BBSR ${ }^{11}$ abgetragen. In besonders dünn besiedelten, ländlichen Kreisen ist das Verhältnis hoch, hier entfallen auf $1,00 \mathrm{~m}^{2}$ Bruttogrundfläche $1,76 \mathrm{~m}^{2}$ Flurstückfläche. In städtischen Kreisen ist das Verhältnis mit 1,00:1,10 deutlich niedriger, während in Großstädten ähnliche Flächenverhältnisse wie in den ländlichen Kreistypen zu verzeichnen sind.

Ursächlich für diese Verteilung könnten einander überlagernde, gegenläufige Effekte sein: Während in ländlichen Kreisen ausreichend Flächen für die Errichtung neuer Warenlagergebäude zur Verfügung stehen, ist in städtischen Kreisen ein höherer Flächendruck feststellbar. Gleichzeitig ist zu vermuten, dass in Großstädten der Fokus stärker auf flächenintensiven Umschlagsimmobilien liegt. Diese oftmals kleineren Gebäude zum reinen Güterumschlag müssen zwingend von mehreren Seiten angedient werden, wodurch das Verhältnis von Bruttogrundfläche zu Flurstückfläche hier höher ausfällt als bei anderen Typen (VeresHomm/Kübler/Weber et al. 2015: 88). Hinzu kommt im Falle Rostocks die Sonderfunktion als überörtlicher Hafenstandort, infolgedessen die Flurstückflächen zur Lagerung - beispielsweise von Baustoffen und anderem Stückgut stärker ins Gewicht fallen als in anderen Städten. Zum Aus-

\footnotetext{
11 https://www.bbsr.bund.de/BBSR/DE/forschung/ raumbeobachtung/Raumabgrenzungen/deutschland/ kreise/siedlungsstrukturelle-kreistypen/kreistypen.html (21.12.2020).
}

gleich dieser Effekte wurde im Folgenden ein Gesamtdurchschnitt aller vier Kreistypenwerte herangezogen.

Abschließend wurden die ermittelten Verhältnisse $\mathrm{V}^{\mathrm{BGF}: \mathrm{NF}}$ und $\mathrm{V}^{\mathrm{BGF}: F F}$ mit den jährlich summierten gemeindescharfen Nutzflächen von Warenlager-Baumaßnahmen des FDZ-Datensatzes multipliziert. Im Ergebnis steht ein deutschlandweiter Überblick über die Flächeninanspruchnahme von Warenlager-Baumaßnahmen des Zeitraums 2000 bis 2015 zur Verfügung. Zur Vergleichbarkeit der Flächeninanspruchnahme gegenüber der verfügbaren Baufläche wurde diesen Daten gemeindescharf die verfügbare Gebäude- und Freifläche (GFF) des Jahres 2015 gegenübergestellt. Auf diesem Weg ist der relative Vergleich der Flächeninanspruchnahme je Gemeinde möglich. Datengrundlagen hierfür sind die Regionaldaten des Statistischen Bundesamtes zur Bodenfläche nach Art der tatsächlichen Nutzung. ${ }^{12}$

Nachfolgend wird ein deskriptiver Überblick über den FDZ-Datensatz gegeben, wobei insbesondere die Entwicklung im Zeitverlauf sowie die Verteilung der Baumaßnahmen im Raum sowie nach Bauherren und Gemeindegrößenklasse im Fokus stehen.

\section{Bestandskonzentration und sektorale Konzentration}

In Deutschland wuchs zwischen 2000 und 2015 die Nutzfläche in Warenlagergebäuden größer $2.500 \mathrm{~m}^{2}$ durch Baumaßnahmen um rund 50,6 Millionen $\mathrm{m}^{2}$ (vgl. Abbildung 5).

Die Fertigstellungen unterliegen im Zeitverlauf starken Schwankungen. So fiel die jährlich fertiggestellte Nutzfläche von rund 3 Millionen $\mathrm{m}^{2}$ im Jahr 2000 auf etwa 2,4 Millionen $\mathrm{m}^{2}$ im Jahr 2004, um innerhalb von vier Jahren um mehr als $70 \%$ auf rund 4 Millionen $\mathrm{m}^{2}$ anzuwachsen. Nach einem kurzen Einbruch der Fertigstellungszahlen in der Zeit nach 2008 stieg die Bautätigkeit bis zum Jahr 2015 erneut auf rund 3,8 Millionen $\mathrm{m}^{2}$. Im Durchschnitt stieg die jährlich fertiggestellte Nutzfläche über alle Erhebungsjahre um $3 \%$ pro Jahr.

Dieser Anstieg ist unter anderem Ausdruck einer deutlich gestiegenen Durchschnittsgröße neu errichteter Warenlagergebäude. Während Warenlagergebäude im Jahr 2000 noch durchschnittlich $6.600 \mathrm{~m}^{2}$ Nutzfläche aufwiesen, stieg die Fläche pro Gebäude nahezu kontinuierlich bis 2015 auf rund $9.000 \mathrm{~m}^{2}$ an - ein klares Anzeichen für Konzentrationseffekte im Sektor. Diese Beobachtung deckt sich mit Er-

\footnotetext{
12 https://www.regionalstatistik.de/genesis//online/ data?operation=table \& code $=33111-01-01-5 \&$ levelindex=1\&levelid=1589541766973 (21.12.2020).
} 


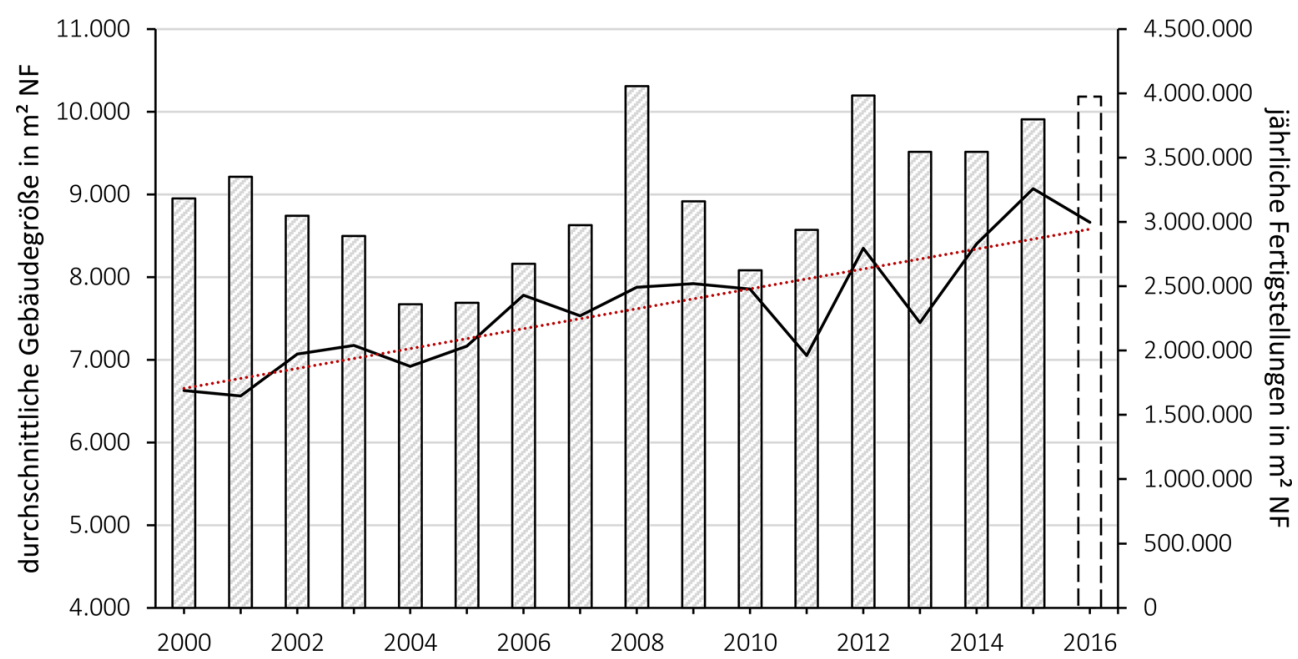

Abbildung 5 Jährliche Fertigstellungen von Warenlagergebäuden in Deutschland in $\mathrm{m}^{2}$ Nutzfläche (Balken, rechte Achse); durchschnittliche Gebäudegröße (Linien, linke Achse) in $\mathrm{m}^{2}$ Nutzfläche Anmerkung: rote Linie = Trendgerade; Werte für 2016 ohne Nachmeldungen Quelle: Statistik der Baufertigstellungen 2000-2016, http://www.forschungsdatenzentrum.de/de/ bauen-und-wohnen/baufertigstellungen (21.12.2020); eigene Berechnungen

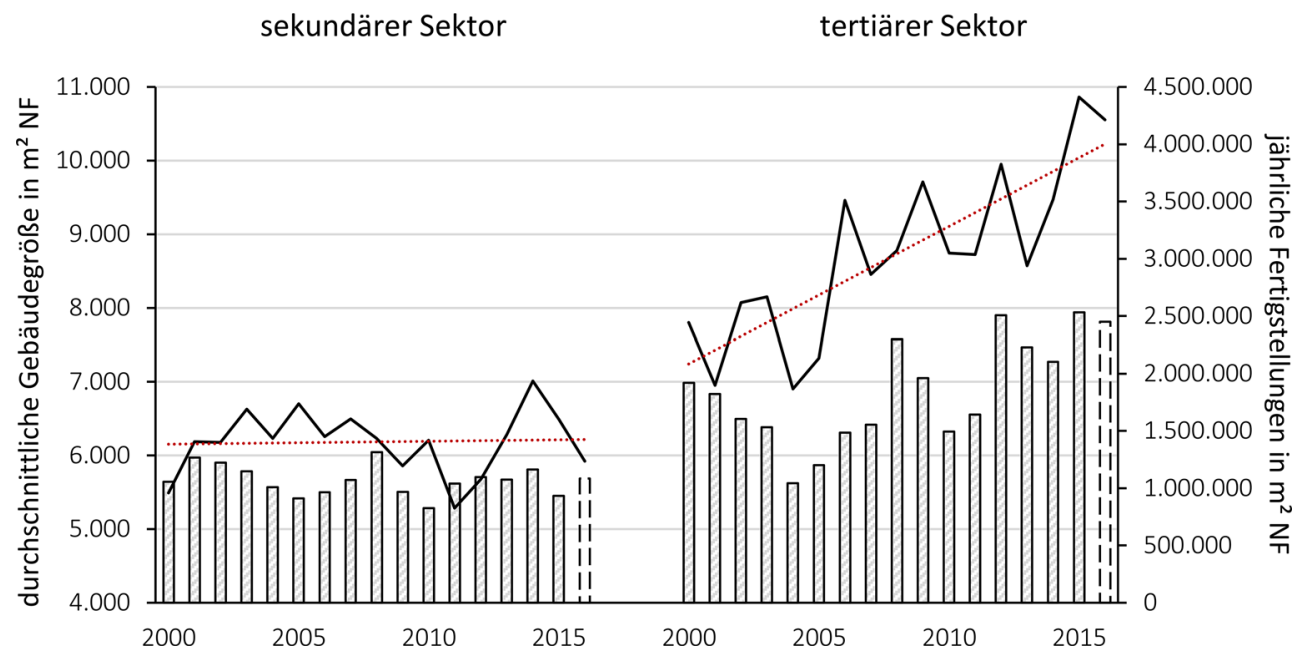

Abbildung 6 Vergleich der jährlichen Fertigstellungen (Balken, rechte Achse) sowie der durchschnittlichen Gebäudegrößen (Linien, linke Achse) zwischen Bauherren im sekundären und tertiären Sektor in $\mathrm{m}^{2}$ Nutzfläche

Anmerkung: rote Linie = Trendgerade; Werte für 2016 ohne Nachmeldungen

Quelle: Statistik der Baufertigstellungen 2000-2016, http://www.forschungsdatenzentrum.de/de/

bauen-und-wohnen/baufertigstellungen (21.12.2020); eigene Berechnungen

hebungen von McKinnon (2009: 295) zum Wachstum der Durchschnittsgröße von Distributionsimmobilien in Großbritannien.

Ähnlich wie in Großbritannien ist auch in Deutschland dieses Größenwachstum maßgeblich auf das Segment der Bauherren des Dienstleistungssektors zurückzuführen (vgl. Abbildung 6). Während die Durchschnittsgröße von Warenlagergebäuden des sekundären Sektors im Zeitverlauf konstant bleibt, wächst die Durchschnittsgröße neu errichteter Gebäude durch Bauherren im tertiären Sektor (Han- del, Gastgewerbe, Finanz-, Versicherungs- und Unternehmensdienstleister, Logistikdienstleister). Hierdurch gewinnt dieser Sektor für die Bautätigkeit von Warenlagergebäuden immer stärker an Bedeutung, während die Bedeutung der Bautätigkeit durch den sekundären Sektor rückläufig ist. Wurden im Durchschnitt der Jahre 2000 bis 2005 etwa $38 \%$ der deutschlandweiten Nutzfläche in Warenlagergebäuden durch das produzierende Gewerbe errichtet, so schrumpfte dessen Anteil zwischen 2006 und 2010 auf 33\% und weiter zwischen 2011 und 2015 auf 29\% (vgl. Abbildung 7). 


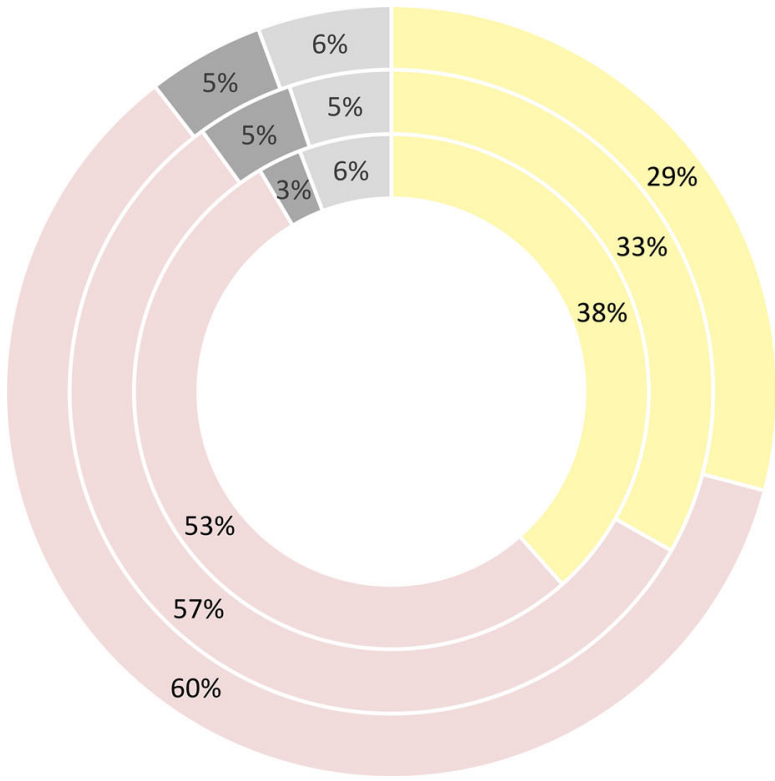

von Innen nach Außen:

$\varnothing 2000-2005$

$\varnothing 2006-2010$

$\varnothing 2011$ - 2015

\section{Sekundär \\ Tertiär \\ - Fonds \\ Sonstige}

Abbildung 7 Vergleich der Baufertigstellungen von Warenlagergebäuden nach Bauherren und Zeiträumen bezogen auf eine durchschnittliche Nutzfläche

Quelle: Statistik der Baufertigstellungen 2000-2016, http://www.forschungsdatenzentrum.de/de/

bauen-und-wohnen/baufertigstellungen (21.12.2020); eigene Berechnungen

In gleichem Ausmaß wuchs der Anteil der Fertigstellungen durch Bauherren des Dienstleistungssektors und von Immobilienfonds.

Die Gründe für diese sektorale Verschiebung der Baufertigstellungen sind vielfältig:

- eine anhaltende Verschiebung des gesamtwirtschaftlichen Schwerpunkts in Deutschland vom verarbeitenden Gewerbe hin zum Dienstleistungsgewerbe,

- die fortschreitende Auslagerung logistischer Dienstleistungen durch das produzierende Gewerbe an spezialisierte Third-party-logistics-Unternehmen (Bowen 2008: 380),

- die Auslagerung der Produktion und damit der Produktionslogistik in Länder mit niedrigeren Produktionskosten,

- der Bedeutungszuwachs des deutschen Online-Handels (Dannenberg/Willkomm/Zehner 2017: 3) und der damit verbundene deutschlandweite Ausbau der Distributionsinfrastruktur durch den Handel (Lengauer/Gierlinger/ Kellermayr-Scheucher et al. 2015: 48),

- in diesem Zeitraum neu entwickelte Logistikpraktiken des Supply-Chain-Managements (Rodrigue 2013) verbunden mit neuen Anforderungen an Lagerhallen und Distributionsimmobilien (Klauser 2012: 87), sowie
- der wachsende Stellenwert des Logistikstandorts Deutschland im internationalen Vergleich (Weltbank 2019 ${ }^{13}$; Veres-Homm/Kübler/Weber et al. 2015: 145).

\section{Räumliche Konzentrationseffekte}

Neben dieser sektoralen Konzentration auf die Dienstleistungsbranche sowie der einhergehenden Bestandskonzentration auf wenige, immer größere Lagergebäude sind auch räumliche Konzentrationseffekte sichtbar. Beim Blick auf die deutschlandweite Verteilung auf Gemeindeebene zeigt sich der große Einfluss des direkten Zugangs zur Autobahn bei der Errichtung neuer Warenlagergebäude (vgl. Abbildungen 8 und 9).

Bis auf wenige Ausnahmen, beispielsweise im industriell geprägten Baden-Württemberg mit seinen vielen historisch gewachsenen mittelständischen Betrieben, haben fast alle Gemeinden mit hohen Baufertigstellungszahlen einen direkten Zugang zur Autobahn. Dieser Zusammenhang verstärkt sich im späteren Betrachtungszeitraum 2008 bis 2015 nochmals. Gleichzeitig ist eine Schwerpunktverschiebung der Bautätigkeit von West nach Ost feststellbar. Im ersten Betrachtungszeitraum wurde die überwiegende Mehrheit der

\footnotetext{
${ }^{13}$ Vgl. https:// lpi.worldbank.org/international/aggregatedranking (21.12.2020).
} 


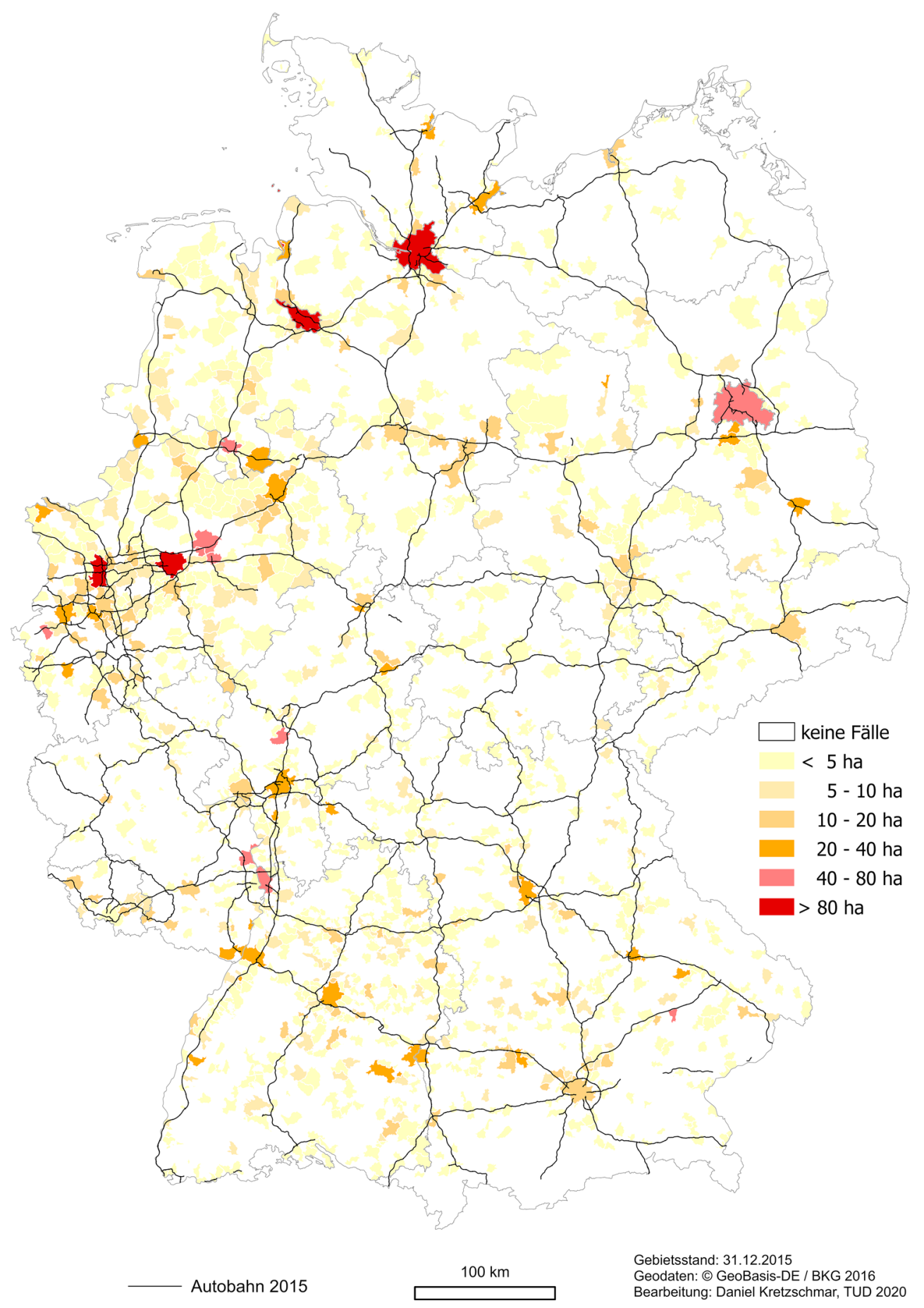

Abbildung 8 Vergleich der Flächeninanspruchnahme der Jahre 2000 bis 2007 auf Gemeindeebene Quelle: Statistik der Baufertigstellungen 2000-2015 http://www.forschungsdatenzentrum.de/de/ bauen-und-wohnen/baufertigstellungen (21.12.2020), Flächenerhebung nach Art der tatsächlichen Nutzung; eigene Berechnungen

Nutzflächen in den Hansestädten Hamburg, Bremen und Dortmund, entlang der Rheinschiene sowie entlang der A5 zwischen Bad Hersfeld und Mannheim sowie der A8 zwischen Karlsruhe und München errichtet. Im zweiten Be- trachtungszeitraum ist die gestiegene Bedeutung der Drehkreuze Leipzig, Erfurt und Nürnberg sowie der Achse Hannover-Wolfsburg-Magdeburg entlang der A2 sichtbar. 


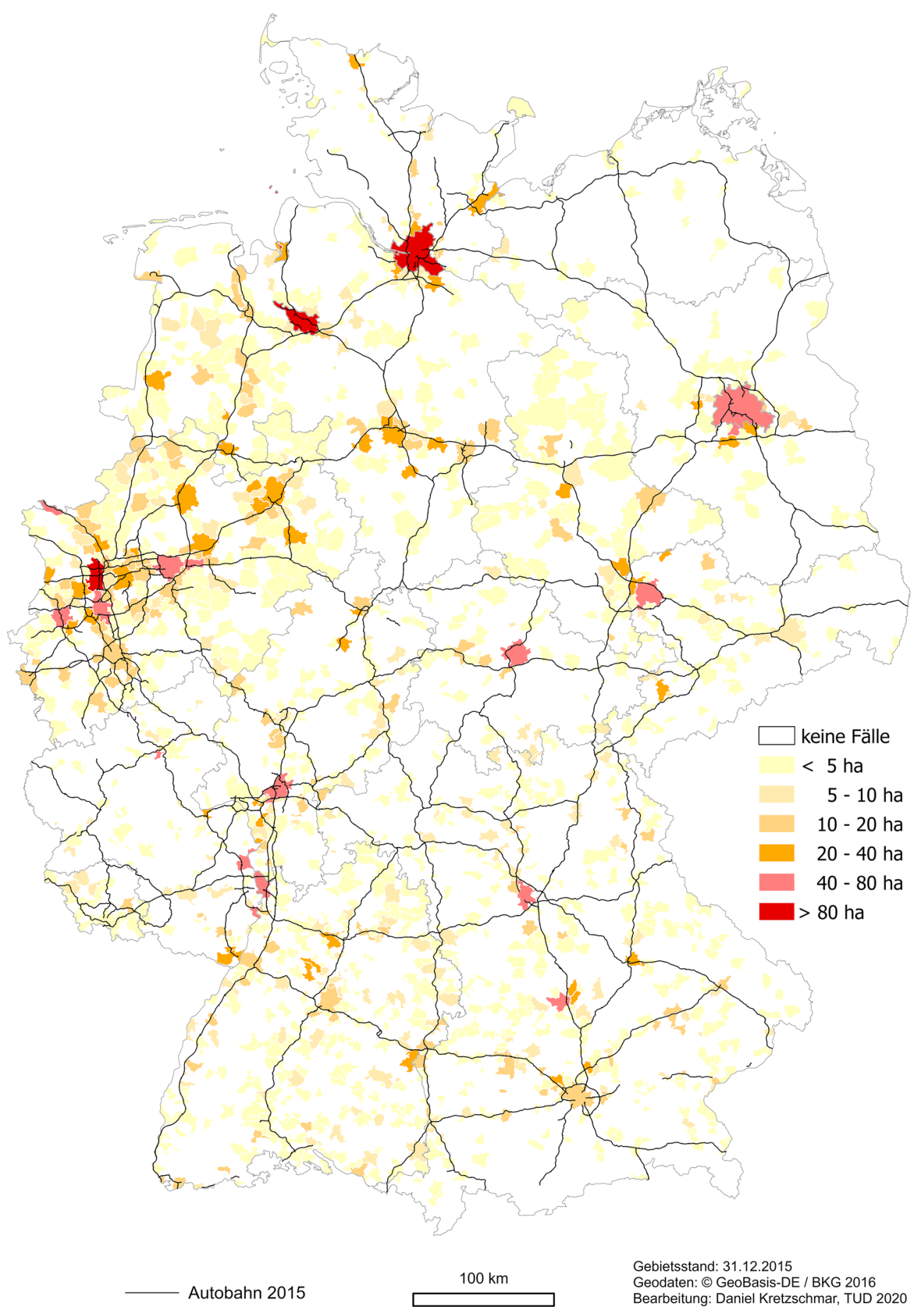

Abbildung 9 Vergleich der Flächeninanspruchnahme der Jahre 2008 bis 2015 auf Gemeindeebene Quelle: Statistik der Baufertigstellungen 2000-2015 http://www.forschungsdatenzentrum.de/de/ bauen-und-wohnen/baufertigstellungen (21.12.2020), Flächenerhebung nach Art der tatsächlichen Nutzung; eigene Berechnungen

Diese räumliche Verlagerung geht einher mit einer Ausweitung der Flächeninanspruchnahme. Lag im ersten Betrachtungszeitraum der Jahre 2000 bis 2005 die tägliche Flächeninanspruchnahme von Warenlagergebäuden in
Deutschland noch bei 2,3 ha/Tag, so wuchs dieser Wert entgegen des allgemeinen Trends auf 2,5 ha/Tag im Zeitraum 2006 bis 2010 und nochmals auf 2,9 ha/Tag im Zeitraum 2011 bis 2015. Die räumliche Verlagerung der Bautätigkeit 


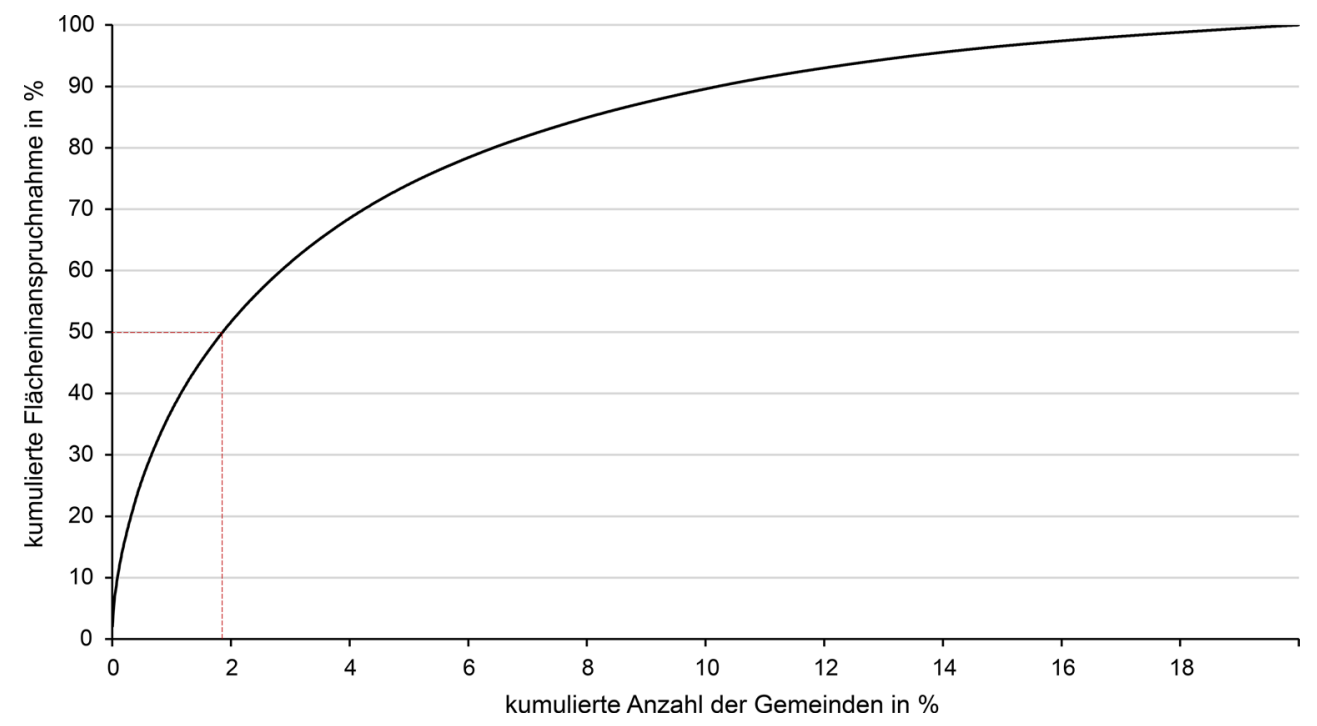

Abbildung 10 Histogramm der Flächeninanspruchnahme durch Warenlagergebäude über alle Gemeinden in Deutschland (in \%), Gemeinden absteigend nach Flächeninanspruchnahme durch Warenlagergebäude sortiert

Quelle: Statistik der Baufertigstellungen 2000-2015 http://www.forschungsdatenzentrum.de/de/

bauen-und-wohnen/baufertigstellungen (21.12.2020); eigene Berechnungen

in den Osten führt demnach nicht zu einer Substitution der Flächeninanspruchnahme im Westen, sie ergänzt und erweitert diese vielmehr.

Trotz der allgemeinen Zunahme der Flächeninanspruchnahme weist der überwiegende Teil der Gemeinden keine Bautätigkeit großer Warenlagergebäude auf (weiße Flächen). Nur in rund $20 \%$ aller Gemeinden ist überhaupt eine Neubautätigkeit feststellbar, wobei in vielen Kommunen wiederum nur ein einziges Gebäude dieser Größenklasse im Zeitraum 2000-2015 errichtet wurde. Die Bautätigkeit konzentriert sich räumlich auf wenige, verkehrlich gut vernetzte Gemeinden.

Sichtbar wird dieser Effekt in Abbildung 10. Hier wurden die Gemeinden mit Bautätigkeit im Warenlagersegment anteilig gegen die durch diese Bautätigkeit in ihnen verursachte Flächeninanspruchnahme abgetragen. 50\% des landesweiten Flächenkonsums von Warenlagergebäuden werden von lediglich 1,8\% aller Gemeinden in Deutschland getragen - eine extreme räumliche Konzentration.

Interessant ist in diesem Zusammenhang eine Differenzierung nach Gemeindegrößenklassen. Es zeigt sich, dass gerade kleine Gemeinden anteilig erhebliche Flächen für den Neubau eines Warenlagers aufbringen müssen. Eine Differenzierung der anteiligen Flächeninanspruchnahme in Abbildung 11 veranschaulicht, dass gerade kleine und kleinste Gemeinden mit weniger als 1.000 Einwohnern große Teile der ihnen zur Verfügung stehenden Flächen durch den Neubau eines Warenlagergebäudes beanspruchen. In Gemeinden mit weniger als 500 Einwohnern führte im Durchschnitt bereits der Bau eines einzigen Wa- renlagergebäudes größer als $2.500 \mathrm{~m}^{2}$ zur Beanspruchung von über $6 \%$ der gemeindeeigenen Gebäude- und Freiflächen im Siedlungsbestand. In extremen Fällen wurden im gesamten Betrachtungszeitraum knapp unter 50\% der 2015 im Siedlungsbestand ausgewiesenen Gebäude- und Freiflächen durch den Neubau von Warenlagergebäuden beansprucht - so beispielsweise in der rheinland-pfälzischen Gemeinde Grolsheim ${ }^{14}$.

\section{Diskussion}

Die hier vorgestellte Methodik erlaubt es erstmals, die Flächeninanspruchnahme von Warenlagergebäuden konkret und regional differenziert zu quantifizieren und zu bewerten. Der überarbeitete Datensatz des Forschungsdatenzentrums (FDZ) ermöglicht detaillierte Analysen der Dynamik im Segment, die sowohl räumlich als auch bezogen auf den sachlichen Teilmarkt sowie den Bauherrn des Gebäudes weiter differenziert werden können. Durch die Übersetzung der Bautätigkeit in geeignete Flächenmaße wären in methodisch gleicher Weise auch Aussagen zum Flächenverbrauch anderer Nutzungsklassen im Nichtwohngebäudesegment möglich.

Die Nutzung allgemeiner Umrechnungsparameter zwi-

\footnotetext{
${ }^{14}$ Die Gemeinde beherbergt Warenlagergebäude zahlreicher großer Logistikunternehmen in Deutschland, darunter Kühne + Nagel, DB Schenker, Rhenus Logistics und CTL Cargo Trans.
} 


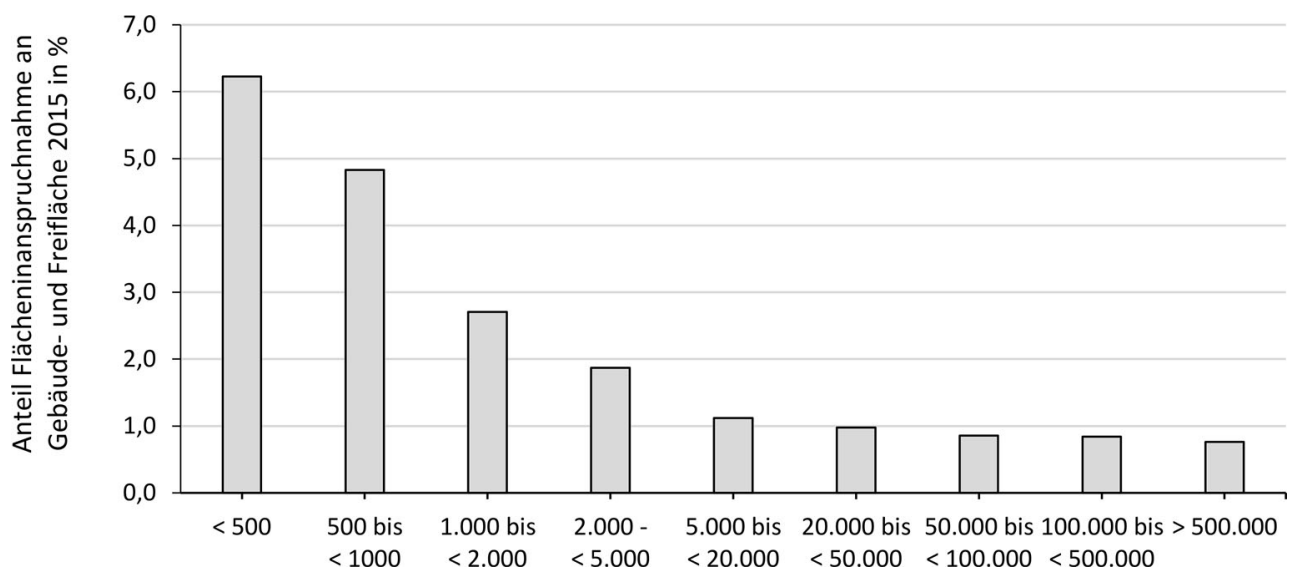

Abbildung 11 Anteil der Flächeninanspruchnahme durch neu errichtete Warenlager an der Gebäudeund Freifläche 2015 (in \%), nach Gemeindegröße je Einwohner

Quelle: Statistik der Baufertigstellungen 2000-2015 http://www.forschungsdatenzentrum.de/de/ bauen-und-wohnen/baufertigstellungen (21.12.2020), Flächenerhebung nach Art der tatsächlichen Nutzung; eigene Berechnungen

schen den Flächenmaßen Nutzfläche, Bruttogrundfläche und Flurstückfläche erlaubt deutschlandweit vergleichbare Ergebnisse, welche zu Lasten der kleinräumigen Genauigkeit gehen. Die Betrachtung der Unterschiede im Verhältnis aus Bruttogrundflächen zu Flurstückflächen in Abbildung 4 - allein auf der Ebene der Kreistypen - macht deutlich, dass die hier vorgestellten Ergebnisse nur einen Anhaltspunkt für die tatsächliche Flächeninanspruchnahme darstellen, die örtlich unterschiedlich belastbar sind. So ermittelten Veres-Homm, Kübler, Weber et al. (2015: 136) bei einer Untersuchung von 2.500 Warenlagergebäuden ein durchschnittliches Verhältnis von Bruttogrundfläche zu Flurstïckfläche von 1,00 m²: 1,41 $\mathrm{m}^{2}$ (zum Vergleich hier ermitteltes Verhältnis: $\left.1,00 \mathrm{~m}^{2}: 1,56 \mathrm{~m}^{2}\right)$. Ein Grund für diese Abweichung könnte in der Wahl MecklenburgVorpommerns als Repräsentant eines eher ländlichen Bundeslandes liegen. Die flächenmäßig großen, größtenteils dünn besiedelten Gemeinden bieten einerseits viel Platz für Logistikflächen, gleichzeitig fließen hierdurch überproportional viele Hafenstandorte in die Analyse ein, die besonders große Flurstückflächen aufweisen. Vorteil der hier vorgestellten Methode ist es, dass die Genauigkeit der Ergebnisse durch das Hinzuziehen lokaler Daten fast beliebig bis zum einzelnen Objekt verbessert werden kann. Für das angestrebte Ziel genereller, deutschlandweiter Aussagen zur Ausprägung der Flächeninanspruchnahme wird das hier vorgestellte Vorgehen als zielführend befunden.

Die ermittelte tägliche Flächeninanspruchnahme von 2,3 ha/Tag der Jahre 2000 bis 2005, 2,6 ha/Tag im Zeitraum 2006 bis 2010 sowie 2,9 ha/Tag zwischen 2010 und 2015 scheint bei Abgleich mit der Statistik der Flächen- inanspruchnahme des Umweltbundesamtes ${ }^{15}$ plausibel. So entfallen am aktuellen Rand des Jahres 2018 rund 32 ha/Tag auf Wohnbau-, Industrie- und Gewerbeflächen sowie Flächen öffentlicher Einrichtungen (ohne Abbauland). ${ }^{16}$ Warenlagergebäude wären nach der hier vorgestellten Erhebung für rund 9\% dieser Flächeninanspruchnahme verantwortlich - trotz deutlicher Ausweitung der Bautätigkeit im Wohnungsbau in den vergangenen Jahren.

Erst in diesem deutschlandweiten Vergleich der Bautätigkeit wird die hohe Konzentration der Dynamik auf einige wenige, infrastrukturell gut erschlossene Gemeinden deutlich, welche die Hauptlast der Flächeninanspruchnahme tragen. Übergeordnete Megatrends wie die kontinuierliche $\mathrm{Zu}-$ nahme des Online-Handels und das scheinbar ungebremste Fortschreiten der Globalisierung sorgen im Zusammenhang mit nationalen Effekten zu einer stetigen Professionalisierung der Logistik. Im Zuge des fortschreitenden Ausbaus der Transportinfrastrukturen sowie der wachsenden Bedeutung des Logistikstandorts Deutschland ist eine sektorale Verschiebung der Bautätigkeit weg vom sekundären Sektor hin zu Bauherren des Dienstleistungssektors zu beobachten. Damit einher geht das ungebremste Wachstum der durchschnittlichen Gebäudegrößen um knapp 36\% in den letzten 15 Jahren. Dieser Dreiklang aus sektoraler, räum-

\footnotetext{
15 https://www.umweltbundesamt.de/daten/flaeche-bodenland-oekosysteme/flaeche/siedlungsverkehrsflaeche (21.12.2020).

${ }^{16}$ Der hier beschriebene Wert stellt einen Durchschnittswert der vergangenen Jahre dar und ist aus diesem Grund nur eingeschränkt mit stichtagsbezogenen Werten der Bautätigkeitsstatistik vergleichbar.
} 
licher und gebäudegrößenbezogener Konzentration erlaubt Rückschlüsse auf die Flächeninanspruchnahme.

Zum einen folgt aus größeren Gebäuden zwangsläufig ein größerer Flächenbedarf - und zwar nicht nur bezogen auf die reinen Bruttogrundflächen, sondern in überproportionaler Weise auch bezogen auf die umgebenden Flurstückflächen, die eine immer schnellere Warenabfertigung für immer größere Lkw-Flotten sicherstellen müssen. Diese Flächen sind durch Stell-, Verkehrs-, Rangier- und Verladeflächen nicht selten vollständig versiegelt, weshalb auf dem Segment der Warenlager ein besonderes Augenmerk in der Flächendiskussion liegen sollte.

Zum anderen scheinen gerade kleine, ländliche Gemeinden besonders stark durch die Flächeninanspruchnahme durch Warenlagergebäude betroffen. Ihre typische Lage im Einflussbereich großer Agglomerationsräume mit günstigem Bauland, niedrigeren Durchschnittslöhnen und einem allgemein attraktiveren Preisniveau für Baudienstleistungen könnten ursächlich sein für die überproportional starke Bautätigkeit in diesen Gemeinden. Die Analyse des Verhältnisses von Bruttogrundflächen zu Flurstückflächen bezogen auf die BBSR-Kreistypen zeigt, dass dünn besiedelte, ländliche Kreise im Durchschnitt den größten Flächenverbrauch je Quadratmeter Bruttogrundfläche der in ihnen errichteten Warenlager aufweisen. In der Folge haben 46 der 100 Gemeinden mit der größten Flächeninanspruchnahme pro Kopf im hier betrachteten Untersuchungszeitraum weniger als 2.000 Einwohner. Bei dieser Feststellung ist zu bedenken, dass die angewandte Bagatellgrenze von $2.500 \mathrm{~m}^{2}$ Nutzfläche je Baumaßnahme das Ergebnis zwangsläufig zulasten kleiner Gemeinden verzerrt.

$\mathrm{Zu}$ guter Letzt führt die schrittweise Konzentration auf Logistikdienstleister des tertiären Sektors zu einer immer weiteren Verbreitung des Distributions-Hubs - jenes Prototyps logistischer Effizienz, welcher dank seiner enormen Flächeninanspruchnahme an peripheren, aber gut vernetzten Knotenpunkten globale Warenströme praktisch ohne zeitliche Unterbrechung in regionale und lokale Warenströme umwandelt (Cidell 2015: 21). Der Siegeszug dieses Warenlagertyps ist eng verknüpft mit der wettbewerbsbedingten Notwendigkeit einer immer schnelleren Zustellung (Jörß/Schröder/Neuhaus et al. 2016: 9). Ermöglicht wird diese Verkürzung durch die exzessive Ausweitung der Versorgungsketten, welche nur durch den Bau immer neuer Zentral- und Zwischenlager aufrechterhalten werden können, denn verkürzte Zustellzeiten sind ,,nur mit einer ausgeprägten Regionalisierung der Distributionsstruktur realisierbar" (Lengauer/Gierlinger/Kellermayr-Scheucher et al. 2015: 48). Die Folgen sind ,logistics sprawl“ (Dablanc/ Ogilvie/Goodchild 2014; Aljohani/Thompson 2016) und eine weiter wachsende Flächeninanspruchnahme.
Hinzu kommt, dass Logistikimmobilien trotz ihrer architektonischen Banalität und ihrer zweifelhaften Umweltimplikationen ein veritables Anlagemodell darstellen. Infolge der Finanzkrise etablierten sich Wirtschaftsimmobilien - zu denen neben Bürogebäuden, Industriegebäuden und Handelsgebäuden unter anderem auch Warenlagergebäude zählen - in einer neuen Welt ungekannt niedriger Zinsen als attraktive Anlagealternativen, insbesondere gegenüber den als vergleichbar sicher geltenden Staatsanleihen (Savills 2017: 9). So lag die Spitzenrendite von Warenlagergebäuden in den letzten Jahren stets höher als vergleichbare Renditen von Büro- bzw. Einzelhandelsgebäuden (CBRE 2019: 1), wobei sich in diesen höheren Renditen auch ein höheres Ausfallrisiko widerspiegelt. In der Folge werden Warenlagergebäude bereits heute zu einem erheblichen Anteil spekulativ - das heißt ohne vorherige Vermietungszusagen - errichtet. Neben diesen Vorteilen für Bauherren und Vermieter hat der Neubau eines großen Warenlagergebäudes auch Vorzüge für die Gemeinden. Gerade für kleinere Orte hat die Errichtung eines großen Warenlagergebäudes verhältnismäßig große, positive Auswirkungen auf den Gemeindeetat. Für diese kleineren Orte an der Autobahn ohne ausreichende industrielle Basis und genügend Arbeitskräfte erscheint der Neubau einer Distributionsimmobilie in Anbetracht leerer Kassen oft alternativlos.

All diese Faktoren führen zu einer einseitigen Betrachtung des Warenlagergebäudes als reines Investitionsobjekt. Kalkuliert wird üblicherweise mit einer durchschnittlichen Lebensdauer von weniger als 20 Jahren. Die Dynamik im Neubau von Logistikimmobilien scheint bisher ungebrochen, doch die Systemgrenzen - zum Beispiel in Bezug auf Knappheiten bei Baumaterialien (EUROCONSTRUCT 2018: 127) - werden bei Fortbestand dieser Entwicklung bereits in wenigen Jahren erreicht werden.

\section{Fazit}

Die vorgestellten Ergebnisse zeigen exemplarisch die Notwendigkeit einer deutlich differenzierteren Betrachtung des deutschlandweiten Flächenverbrauchs. Die Bautätigkeit im Ein- und Zweifamilienhaussegment wird im Spannungsfeld zwischen ökologischen Erfordernissen und den Wohnwünschen der Haushalte selbstverständlich auch im Hinblick auf die Flächeninanspruchnahme rege diskutiert. Währenddessen ist die hohe Dynamik einzelner Segmente des Nichtwohnbaus und ihrer flächenmäßigen Bedeutung bestenfalls anekdotisch beschrieben. Die reinen Dimensionen dieser Flächeninanspruchnahme - im Mittel immerhin rund 2,6 ha pro Tag ohne Straßen, bei steigender Tendenz - würden eine größere Resonanz vermuten lassen. Ursächlich für die geringe Aufmerksamkeit waren in der Vergangenheit 
fehlende Daten zur Bautätigkeit sowie ein allgemein geringes öffentliches Interesse an diesen kollektiv verdrängten „Nicht-Orte[n]“ (Klauser 2012: 87).

Eine weitere Ursache dieser Verdrängung aus der öffentlichen Wahrnehmung liegt in der deutschlandweiten Verteilung der Bautätigkeit. Hier zeigt sich ein klarer Fokus auf kleinen, peripher gelegenen Gemeinden entlang der $\mathrm{Au}$ tobahn sowie Gemeinden im unmittelbaren Umfeld großer Agglomerationen. Im Gegensatz zu anderen Nutzungsklassen im Nichtwohnbau, beispielsweise im Segment der Bürogebäude, scheinen Warenlagergebäude in weit höherem Maße in diesen kleinen Gemeinden errichtet zu werden. Da diesen Gemeinden im Mittel geringere Flächenreserven zur Verfügung stehen, sorgt bereits der Bau eines einzigen Warenlagergebäudes zur Beanspruchung von rund $6 \%$ der gemeindeintern verfügbaren Gebäude- und Freiflächenbestände. Der verursachte Flächenverbrauch ist gerade bezüglich einer sinkenden Gesamtnutzungsdauer im Warenlagersegment bedenklich. Vor 20 Jahren errichtete Logistikimmobilien entsprechen in Bezug auf Größe und Ausstattung der Hallen nicht mehr heutigen Anforderungen. Die aus dieser sinkenden Lebensdauer resultierende Beschleunigung der Bestandsdynamik im Wechselspiel von Abriss und Neubau hat direkte Folgen für die Flächeninanspruchnahme in diesen Gemeinden.

Dabei ist strukturpolitisch die deutschlandweite Konzentration der Warenlagergebäude auf wenige Gemeinden im Hinblick auf verkehrsinduzierte Umweltimplikationen und das Landschaftsbild grundsätzlich zu begrüßen. Problematisch ist vielmehr die im Zeitverlauf feststellbare Standorterweiterung, vor allem in Mittel- und Ostdeutschland, welche bestehende Bestände nicht substituiert, sondern erweitert. Im Zusammenspiel mit einem bis dato ungebrochenen Ausbau der Verkehrsinfrastruktur verfestigt sich ein problematischer Zyklus: logistische Ansiedlungen in der Peripherie nehmen neue Autobahntrassen bereits vorweg, neue Autobahnstrecken werden errichtet, wodurch weitere lukrative Warenlagerstandorte erschlossen werden. Dies führt zu einer noch stärkeren verkehrlichen Beanspruchung, woraus die erneute Notwendigkeit zusätzlicher Verkehrstraßen resultiert. Eine Durchbrechung dieses Zyklus ist gegenwärtig nicht in Sicht.

In der Folge ist die indirekte Flächeninanspruchnahme durch Warenlagergebäude größer als in dieser Analyse dargestellt. Wie keine andere Nutzungsklasse induziert ein Warenlagergebäude Verkehr und dadurch die Notwendigkeit einer guten infrastrukturellen Anbindung. Diese Straßenflächen konnten in der hier vorgestellten Analyse - mit Ausnahme der Rangier-, Verlade- und Halteflächen unmittelbar auf den Flurstücken - nicht berücksichtigt werden. Die indirekt durch Warenlagergebäude induzierte Flächeninan- spruchnahme dürfte weit größer ausfallen als hier beschrieben.

Es scheint daher empfehlenswert, dem Warenlagersegment gerade bezogen auf das 30-ha-Ziel der Bundesregierung und des immer noch postulierten Ideals der Innenentwicklung weit stärker als bisher Beachtung zu schenken. Die hier vorgestellte Analyse zeigt, dass es ratsam scheint, in der Flächenhaushaltspolitik stärker nach spezifischen Nutzungsarten, Raumkontexten sowie damit verbundenen Einflussgrößen zu differenzieren. Zu diesem Zweck sollte in einer auf der hier vorgestellten Methodik aufbauenden Analyse die Flächeninanspruchnahme der Bautätigkeit in anderen sachlichen Teilmärkten des Nichtwohngebäudebestandes quantifiziert werden. Zukünftige Beiträge sollten sich in diesem Kontext auf Bürogebäude und Industriegebäude konzentrieren.

Danksagung Herzlicher Dank geht an die Kollegen des Leibniz-Instituts für ökologische Raumentwicklung (IÖR), André Hartmann sowie Martin Schorcht für die Unterstützung bei der Aufbereitung der LoD1Daten sowie an Ulrich Schumacher für die Bereitstellung der Kartengrundlage.

\section{Literatur}

Aljohani, K.; Thompson, R. G. (2016): Impacts of logistics sprawl on the urban environment and logistics: Taxonomy and review of literature. In: Journal of Transport Geography 57, 255-263. https://doi.org/10.1016/j. jtrangeo.2016.08.009

Allen, J.; Browne, M.; Cherrett, T. (2012): Investigating relationships between road freight transport, facility location, logistics management and urban form. In: Journal of Transport Geography 24, 45-57. https://doi.org/10.1016/ j.jtrangeo.2012.06.010

Baker, P. (2004): Aligning Distribution Center Operations to Supply Chain Strategy. In: The International Journal of Logistics Management 15, 1, 111-123. https://doi.org/ 10.1108/09574090410700266

Becqué, R.; Mackres, E.; Layke, J.; Aden, N.; Liu, S.; Managan, K.; Nesler, C.; Mazur-Stommen, S.; Petrichenko, K.; Graham, P. (2016): Accelerating building efficiency: Eight actions for urban leaders. Washington, DC.

BKI - Baukosteninformationszentrum (2010): BKI Baukosten 2010. Statistische Kostenkennwerte für Gebäude. Stuttgart.

BKI - Baukosteninformationszentrum (2012): BKI Baukosten Gebäude 2012. Statistische Kostenkennwerte Teil 1. Stuttgart.

BKI - Baukosteninformationszentrum (2013): BKI Baukosten Gebäude 2013. Statistische Kostenkennwerte Teil 1. Stuttgart.

BKI - Baukosteninformationszentrum (2015): BKI Baukos- 
ten 2015 Neubau. Statistische Kostenkennwerte für Gebäude. Stuttgart.

Bowen, J. T. (2008): Moving places: the geography of warehousing in the US. In: Journal of Transport Geography 16, 6, 379-387. https://doi.org/10.1016/j.jtrangeo.2008. 03.001

Bradley, P.; Kohler, N. (2007): Methodology for the survival analysis of urban building stocks. In: Building Research and Information 35, 5, 529-542. https://doi.org/10.1080/ 09613210701266939

Bulwiengesa (2017): Logistik und Immobilien 2017. Citylogistik. Mit neuen Ideen durch die Stadt. München.

Bulwiengesa (2018): Logistik und Immobilien 2018. Jenseits des Hypes. Das „Brot- und Buttergeschäft“ der Logistik. München.

CBRE (2019): Deutschlands Gewerbe Investmentmarkt, H1 2019. Produktmangel bremst Transaktionsvolumen. Frankfurt am Main.

Cidell, J. (2010): Concentration and decentralization: the new geography of freight distribution in US metropolitan areas. In: Journal of Transport Geography 18, 3, 363371. https://doi.org/10.1016/j.jtrangeo.2009.06.017

Cidell, J. (2015): Distribution centers as distributed places: Mobility, infrastructure and truck traffic. In: Birtchnell, T.; Savitzky, S.; Urry, J. (Hrsg.): Cargomobilities. Moving Materials in a Global Age. New York, 17-34.

Dablanc, L.; Ogilvie, S.; Goodchild, A. (2014): Logistics Sprawl. Differential Warehousing Development Patterns in Los Angeles, California, and Seattle, Washington. In: Transportation Research Record: Journal of the Transportation Research Board 2410, 1, 105-112. https://doi. org/10.3141/2410-12

Dablanc, L.; Ross, C. (2012): Atlanta: a mega logistics center in the Piedmont Atlantic Megaregion (PAM). In: Journal of Transport Geography 24, 432-442. https://doi.org/ 10.1016/j.jtrangeo.2012.05.001

Dannenberg, P.; Willkomm, M.; Zehner, K. (2017): Einzelhandel und räumliche Entwicklung - aktuelle Dynamiken in Deutschland. In: Dannenberg, P.; Willkomm, M.; Zehner, K. (Hrsg.): Einzelhandel in Deutschland. Aktuelle Dynamiken im Kontext neuer Rahmenbedingungen. Mannheim, 1-8. = Geographische Handelsforschung 25.

DBEIS - Department for Business, Energy and Industrial Strategy (2016): Building Energy Efficiency Survey, 2014-15. Overarching Report. London.

EUROCONSTRUCT (2018): 86th EUROCONSTRUCT Summary Report. European Construction: Market Trends until 2021. 86th EUROCONSTRUCT Conference. 2223 November 2018, Paris, France. o.O.

Green Leigh, N.; Hoelzel, N. Z. (2012): Smart Growth's Blind Side. In: Journal of the American Planning Asso- ciation 78, 1, 87-103. https://doi.org/10.1080/01944363. 2011.645274

Hall, P.V.; Hesse, M. (2013): Reconciling cities and flows in geography and regional studies. In: Hall, P.V.; Hesse, M. (Hrsg.): Cities, Regions and Flows. Abingdon, 3-20.

Hassler, U. (2009): Long-term building stock survival and intergenerational management: the role of institutional regimes. In: Building Reseach and Information 37, 5-6, 552-568. https://doi.org/10.1080/09613210903189533

He, M.; Shen, J.; Wu, X.; Luo, J. (2018): Logistics Space: A Literature Review from the Sustainability Perspective. In: Sustainability 10, 8, 2815. https://doi.org/10.3390/ su 10082815

Hesse, M.; Rodrigue, J.-P. (2004): The transport geography of logistics and freight distribution. In: Journal of Transport Geography 12, 3, 171-184. https://doi.org/10.1016/ j.jtrangeo.2003.12.004

IEA - International Energy Agency (2018): 2018 Global Status Report: Towards a zero-emission, efficient and resilient buildings and construction sector. London.

Isaacs, N.; Hills, A. (2014): Understanding the New Zealand non-domestic building stock. In: Building Research and Information 42, 1, 95-108. https://doi.org/10.1080/ 09613218.2013.831274

Jakubicek, P.; Woudsma, C. (2011): Proximity, land, labor and planning? Logistics industry perspectives on facility location. In: Transportation Letters: The International Journal of Transportation Research 3, 3, 161-173. https:// doi.org/10.3328/TL.2011.03.03.161-173

Jenks, C. W.; Jencks, C. F.; Rogers, W. C.; Thomas, C.; Delaney, E. P.; Freer, H. (2012): Preserving and Protecting Freight Infrastructure and Routes Washington, DC. = National Cooperative Freight Research Program Reports 16 .

Jörß, M.; Schröder, J.; Neuhaus, F.; Klink, C.; Mann, F. (2016): Parcel delivery. The future of last mile. Travel, Transport and Logistics. o.O.

Kienzler, H.-P.; Altenburg, S.; Esser, K.; Kurte, J.; Wittowsky, D.; Konrad, K.; van der Vlugt, A.-L.; Groth, S. (2018): Verkehrlich-Städtebauliche Auswirkungen des Online-Handels. Endbericht, Stand 16.08.2018. Bonn.

Kille, C.; Nehm, A. (2018): Zukunft der Logistikimmobilien und Standorte aus Nutzersicht. Mythen. Moden. Trends. Nürnberg.

Klauser, W. (2012): Landschaften der Risikogesellschaft. In: ARCH+ Zeitschrift für Architektur und Städtebau 205, 86-89.

Kohler, N.; Steadman, P.; Hassler, U. (2009): Research on the building stock and its applications. In: Building Research and Information 37, 5-6, 449-454. https://doi.org/ 10.1080/09613210903189384

Kretzschmar, D.; Schiller, G.; Weitkamp, A. (2019): Nicht- 
wohngebäude in Deutschland - Typisierung eines dynamischen Marktes. In: Zeitschrift für Geodäsie, Geoinformation und Landmanagement 144, 3, 157-166. https:// doi.org/10.12902/zfv-0250-2019

Kumar, I.; Zhalnin, A.; Kim, A.; Beaulieu, L. J. (2017): Transportation and logistics cluster competitive advantages in the U.S. regions: A cross-sectional and spatiotemporal analysis. In: Research in Transportation Economics 61, 25-36. https://doi.org/10.1016/j.retrec.2016.07. 028

Lengauer, E.; Gierlinger, D.; Kellermayr-Scheucher, M.; Koll, O.; Kreuzer, M.; Herry, M.; Sedlacek, N. (2015): eComTraf. Auswirkungen von E-Commerce auf das Gesamtverkehrssystem. Wien.

Manner-Romberg, H.; Müller-Steinfahrt, U. (2017): Marktuntersuchung und Entwicklungstrends von Kurier-, Express- und Paketdienstleistungen 2017. Gutachten. Hamburg.

McKinnon, A. (2009): The present and future land requirements of logistical activities. In: Land Use Policy 26, Supplement 1, 293-301. https://doi.org/10.1016/j. landusepol.2009.08.014

Murphy, A. J. (2003): (Re)Solving Space and Time: Fulfilment Issues in Online Grocery Retailing. In: Environment and Planning A: Economy and Space 35, 7, 11731200. https.//doi.org/10.1068/a35102

Nehm, A.; Veres-Homm, U. (2018): Standortkompass. Flächen- und Beschäftigungspotentiale in den deutschen Logistikregionen. Weiterstadt.

Ortlepp, R.; Gruhler, K.; Schiller, G. (2016): Material stocks in Germany's non-domestic buildings: a new quantification method In: Building Research and Information 44, 8, 840-862. https://doi.org/10.1080/09613218.2016. 1112096

Rein, S. (2016): Datenbasis zum Gebäudebestand. Zur Notwendigkeit eines besseren Informationsstandes über die Wohn- und Nichtwohngebäude in Deutschland. Bonn. = BBSR-Analysen Kompakt 09/2016.
Rodrigue, J.-P. (2013): Supply chain management, logistics changes and the concept of friction. In: Hall, P. V.; Hesse, M. (Hrsg.): Cities, Regions and Flows. Abingdon, 58-74. Savills (2017): European Logistics. Warehousing the future. London.

Statistisches Bundesamt (2010): Signierschlüsselverzeichnis für Nichtwohngebäude. Wiesbaden.

Statistisches Bundesamt (2014): Signierschlüssel für Nichtwohngebäude. Redaktionell überarbeitete Fassung. Wiesbaden.

Statistisches Bundesamt (2020): Bauen und Wohnen 2019. Baugenehmigungen / Baufertigstellungen von Nichtwohngebäuden (Neubau). Lange Reihen z.T. ab 1980. Wiesbaden.

UN - United Nations Environment Programme; IEA - International Energy Agency (2017): Towards a zero-emission, efficient, and resilient buildings and construction sector. Global Status Report 2017. New York.

Veres-Homm, U.; Kübler, A.; Weber, N.; Cäsar, E. (2015): Logistikimmobilien - Markt und Standorte 2015. Nürnberg.

Verhetsel, A.; Kessels, R.; Goos, P.; Zijlstra, T.; Blomme, N.; Cant, J. (2015): Location of logistics companies: a stated preference study to disentangle the impact of accessibility. In: Journal of Transport Geography 42, 110121. https://doi.org/10.1016/j.jtrangeo.2014.12.002

Waldheim, C.; Berger, A. (2012): Logistiklandschaften. In: ARCH+ Zeitschrift für Architektur und Städtebau 205, 76-83.

Weltbank (2019): International LPI. Global Rankings 2018. https://lpi.worldbank.org/international/global (15.02.2021).

Woudsma, C.; Jensen, J. F.; Kanaroglou, P.; Maoh, H. (2008): Logistics land use and the city: A spatial-temporal modeling approach. In: Transportation Research Part E: Logistics and Transportation Review 44, 2, 277-297. https://doi.org/10.1016/j.tre.2007.07.006 